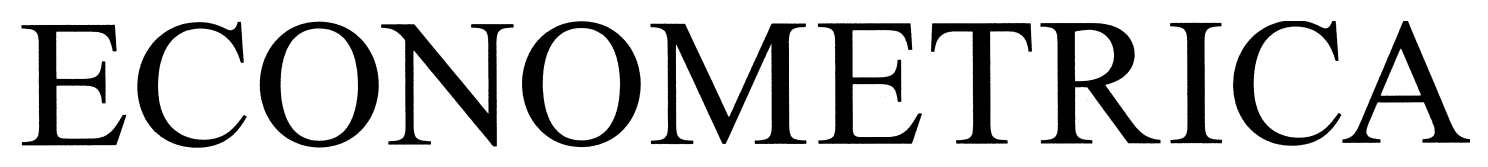

JOURNAL OF THE ECONOMETRIC SOCIETY

An International Society for the Advancement of Economic

Theory in its Relation to Statistics and Mathematics

http://www.econometricsociety.org/

Econometrica, Vol. 87, No. 3 (May, 2019), 837-865

THE PROBABILITY TO REACH AN AGREEMENT AS A FOUNDATION FOR AXIOMATIC BARGAINING

LORENZO BASTIANELLO

LEMMA, Université Paris 2 Panthéon-Assas

MARCO LICALZI

Dept. of Management, Università Ca' Foscari Venezia

The copyright to this Article is held by the Econometric Society. It may be downloaded, printed and reproduced only for educational or research purposes, including use in course packs. No downloading or copying may be done for any commercial purpose without the explicit permission of the Econometric Society. For such commercial purposes contact the Office of the Econometric Society (contact information may be found at the website http://www.econometricsociety.org or in the back cover of Econometrica). This statement must be included on all copies of this Article that are made available electronically or in any other format. 


\title{
THE PROBABILITY TO REACH AN AGREEMENT AS A FOUNDATION FOR AXIOMATIC BARGAINING
}

\author{
LORENZO BASTIANELLO \\ LEMMA, Université Paris 2 Panthéon-Assas \\ MARCO LiCALZI \\ Dept. of Management, Università Ca' Foscari Venezia
}

\begin{abstract}
We revisit the Nash bargaining model and axiomatize a procedural solution that maximizes the probability of successful bargaining. Our characterization spans several known solution concepts, including the special cases of the Nash, egalitarian, and utilitarian solutions. Using a probability-based language, we offer a natural interpretation for the product operator underlying the Nash solution: when the bargainers' individual acceptance probabilities are independent, their product recovers the joint acceptance probability.
\end{abstract} las.

KEYWORDS: Cooperative bargaining, mediation, arbitration, benchmarking, copu-

\section{INTRODUCTION}

THE AXIOMATIC FOUNDATIONS of two-person bargaining theory laid out by Nash (1950) stand out as a cornerstone for simplicity and elegance. They have spawned a huge body of literature; see Thomson (1994). But there is something amiss among their many successful applications.

The Nash solution recommends to pick an outcome that maximizes the product of bargainers' utilities. Many economic models turn this precept into a shortcut for predicting how unstructured bargaining will be resolved, but hardly anybody uses it in real situations. One reason for this disconnect is that the Nash solution "lacks a straightforward interpretation since the meaning of the product of two von Neumann-Morgenstern utility numbers is unclear" (Rubinstein, Safra, and Thomson, 1992, p. 1172). It is difficult to advocate a solution that the bargainers cannot make sense of.

We revisit the Nash model and address this difficulty, switching from a utility-based language to a probability-based language. We assume that bargainers have ordinal preferences over physical alternatives, relaxing the (covert) requirement that they also rank lotteries among alternatives. Next, we introduce uncertainty over which alternatives bargainers are willing to accept. Then we illustrate and axiomatize a solution that selects an alternative to maximize the probability that the bargainers reach an agreement. This procedure encompasses the standard Nash solution and provides a sound prescriptive advice in real situations.

An example may be useful to illustrate our approach. Two agents bargain over a set $A$ of feasible alternatives, described in physical terms. Assume that $A$ is a nonempty, compact,

Lorenzo Bastianello: lorenzo.bastianello@u-paris2.fr

Marco LiCalzi: licalzi@unive.it

This paper subsumes an earlier paper titled "Target-based solutions for Nash bargaining." Some ideas herein were first presented at a workshop on the occasion of Castagnoli's 70th birthday. We thank F. Dietrich, F. Durante, M. Marinacci, W. Trockel, and audiences at Bielefeld, Cergy-Pontoise, IMPA, INSPER, Padua, Paris 1, Paris School of Economics, Salento, Salerno, and Turin for their comments. We are grateful for the referees' contribution and for the editorial stewardship. LiCalzi acknowledges financial support from the European Union's Horizon 2020 research and innovation programme under Grant Agreement no. 732942. 
and convex subset of $\mathbb{R}^{n}$. Each bargainer $i=1,2$ has an ordinal continuous preference $\succsim_{i}$ over $A$. The two agents hire a mediator to recommend a solution and help them reach an agreement. The agents' ordinal preferences are commonly known, but the mediator does not know what it takes for an agent to accept a proposal $a$ from $A$.

More formally, suppose that $i=1,2$ accepts a proposal $a$ if and only if $a \succsim_{i} t_{i}$, where $t_{i}$ in $A$ is $i$ 's acceptance threshold or, for short, his target. The mediator has incomplete information about the bargainers' targets: she believes that each target is a random variable $T_{i}$ taking values in $A$. For any proposal $a$, her beliefs give a pair of individual acceptance probabilities $\left(p_{1}, p_{2}\right)$, where $p_{i}=P\left(a \succsim_{i} T_{i}\right)$. If she also believes that the two targets are stochastically independent, the probability that both accept $a$ is the product $p_{1} \cdot p_{2}$ of the individual acceptance probabilities.

The mediator can recommend any feasible alternative, but she cannot impose it: if she suggests $a$, it is left to the bargainers to accept it. Her goal is to find a proposal that maximizes the probability that the agents consent to it: if she believes that the bargainers' targets are stochastically independent, she should advance a proposal $a$ that maximizes the product $p_{1} \cdot p_{2}$. In this example, we interpret the Nash solution as a rule for the mediator: maximize the probability that the bargainers reach an agreement, given that their targets are private information and independently distributed. From a prescriptive viewpoint, the mediator may use $p_{1} \cdot p_{2}$ to construct a ranking over alternatives consistent with bargainers' ordinal preferences, and she can provide a clear-cut argument for her recommendation.

Moving beyond the Nash product, we characterize the general case where individual acceptance probabilities are aggregated under an arbitrary dependence structure. In particular, we recover axiomatizations for the special cases of the egalitarian and the utilitarian solutions. This offers a unifying interpretation for several solution concepts, up to which dependence structure is presumed.

A switch from a utility-based language to a probability-based language carries modeling advantages. Consider the special case where the mediator is to propose a division for a homogeneous cake of unit size, by offering a portion of size $x_{1}=x$ to $i=1$ and the complementary portion of size $x_{2}=1-x$ to $i=2$. Then $A=\{(x, 1-x): x \in[0,1]\}$. Suppose that each bargainer has preferences increasing in his own portion of the cake. Given a proposal $a=\left(x_{1}, x_{2}\right)$ and the mediator's beliefs on $i$ 's target $T_{i}$, she assesses $i$ 's acceptance probability by the distribution function $F_{i}\left(x_{i}\right)=P\left(x_{i} \succsim_{i} T_{i}\right)$. If bargainers' targets are stochastically independent, she maximizes the probability of joint acceptance by solving

$$
\max _{x \in[0,1]} F_{1}(x) F_{2}(1-x) .
$$

The Nash model presumes instead that it is commonly known that each bargainer has EU-preferences and (after a suitable normalization) solves

$$
\max _{x \in[0,1]} u_{1}(x) u_{2}(1-x) .
$$

There is a formal analogy between the two problems, but Nash's approach abstracts away all the particulars except for the bargainers' risk attitudes. If the agents are risk-neutral and the cake is worth 1 euro to the first one and $v$ euro to the second one, the Nash solution prescribes the same division whether $v=1$ or $v=1000$. The mediator, on the other hand, formulates her beliefs with respect to a specific bargaining problem: she can assess 
different distributions for the individual targets depending on $v=1$ or $v=1000$. A mediator who acknowledges that the value $v$ affects bargainers' targets comes to different recommendations.

The utility-based literature offers two alternative interpretations for the product operator. First, Trockel (2008) viewed the Nash product as a social welfare function that aggregates the two bargainers' (normalized) utilities into a social ranking and evaluates a pair $\mathbf{u}=\left(\mathbf{u}_{1}, \mathbf{u}_{2}\right)$ by the Lebesgue measure of the set of utility pairs that are Paretodominated by $\mathbf{u}$. In our approach, the product aggregates the two individual acceptance probabilities into a social (or mediator's) ranking, but the evaluation is interpreted as the probability of joint acceptance.

Second, Roth (1979, Section I.C) framed the bargaining model as a single-person decision problem, where $i$ chooses a claim that maximizes his expected utility under the belief that the utility demanded by the other bargainer $j=3-i$ is uniformly distributed; see also Anbar and Kalai (1978). Then the Nash solution emerges from the independent choices of the two bargainers. From a game-theoretic viewpoint, Roth noted that juxtaposing the bargainers' decision problems makes their expectations mutually inconsistent. But our mediator deals with a genuine single-person decision problem where her goal is to maximize the probability of success with respect to her own beliefs.

Section 2 describes our framework and traces it back to Nash (1950). Section 3 characterizes the probability-based Nash solution. Section 4 axiomatizes the class of solutions that maximize the probability to reach an agreement; moreover, we characterize the egalitarian and utilitarian solutions as special cases. Section 5 formalizes a benchmarking procedure to align the incomplete information of an agent with the ordinal preferences of a bargainer and discuss its implications for the utility-based Nash model. Section 6 reviews the relationships of our approach with Nash bargaining. Section 7 offers a commentary and a few additional results. Technicalities and proofs are relegated to the Appendix.

\section{THE FRAMEWORK}

The bargaining problem in Nash (1950, p. 155; emphasis added) considers a situation where two parties may cooperate to their mutual benefit, but "no action taken by one of the individuals without the consent of the other can affect the well-being of the other one." Thus, either agent is able to enforce a default outcome. Nash postulated "that the two individuals are highly rational, that each can accurately compare his desires for various things, that they are equal in bargaining skill, and that each has full knowledge of the tastes and preferences of the other." Then he argued that "a theoretical treatment of bargaining situations [abstracts] from the situation to form a mathematical model in terms of which to develop the theory." Our framework complies with these desiderata.

There is a set $A$ of available (physical) alternatives, including a default outcome $\delta$ that occurs if bargaining breaks down. We assume that $A$ is a subset of a topological space, endowed with the relative topology. Each bargainer $i=1,2$ has a continuous ${ }^{1}$ preference relation $\succsim_{i}$ on $A$; thus, he has a most preferred element for each compact subset of $A$.

The quadruple $\left(A, \delta ; \succsim_{1}, \succsim_{2}\right)$ is a bargaining problem with ordinal preferences. It is a modest but uncontroversial assumption that any alternative $a^{*}$ associated with a solution to this problem should be:

feasible: $a^{*} \in A$;

\footnotetext{
${ }^{1}$ A preference relation is continuous on $A$ if, for any $a \in A$, the set $W(a)=\{b \in A: b \prec a\}$ of strictly worse alternatives and the set $B(a)=\{b \in A: b \succ a\}$ of strictly better alternatives are open.
} 
individually rational: $a^{*} \succsim_{i} \delta$ for $i=1,2$;

Pareto optimal: there is no $a$ in $A$ such that $a \succsim_{i} a^{*}$ for $i$ and $a \succ_{j} a^{*}$ for $j=3-i$.

Individual rationality recognizes that no rational bargainer would consent to an outcome strictly worse than what he can unilaterally enforce. Pareto optimality prevents wasting any opportunity to benefit an agent without harming the other one.

Let $A^{*}$ be the set of feasible alternatives that are individually rational and Pareto optimal for the bargaining problem $\left(A, \delta ; \succsim_{1}, \succsim_{2}\right)$. Clearly, $i$ and $j$ have opposing preferences on $A^{*}$. We assume that $A^{*}$ is compact so each bargainer $i$ has a best choice $M_{i}$ in $A^{*}$. Without loss of generality, let $M_{i}$ be unique and $M_{1} \neq M_{2}$. Then $M_{i} \succ_{i} M_{j}$ for $j=3-i$ and $M_{j}$ is the worst choice in $A^{*}$ for $i$. All of the above is commonly known to the bargainers and to any third party-for example, a mediator.

A (nonempty) subset of $A^{*}$ is a solution. When it can be rationalized as the set of maximal outcomes with respect to a complete ranking on $A$, we say that it is a procedural solution. This paper is concerned with procedural solutions.

Nash (1950) refined the bargaining problem with ordinal preferences by adding the assumption that bargainers maximize expected utility. ${ }^{2}$ It is common knowledge that each bargainer has expected utility preferences over the set of lotteries on $A$, with a von Neumann-Morgenstern utility $u_{i}: A \rightarrow \mathbb{R}$ consistent with $\succsim_{i}$ and unique up to increasing affine transformations. For short, we say that the two bargainers have $E U$ preferences based on the (vNM-)utility functions $u_{1}, u_{2}$.

The solution axiomatized by Nash concerns the smaller class of bargaining problems with EU-preferences $\left(A, \delta ; u_{1}, u_{2}\right)$. The Nash solution is procedural, because it is rationalized by the ranking associated with the product

$$
\left[u_{1}(a)-u_{1}(\delta)\right] \cdot\left[u_{2}(a)-u_{2}(\delta)\right] .
$$

This paper demonstrates a more general approach to refine ordinal preferences and uses it to derive procedural solutions. The key technical step in Nash's treatment relies on the expected utility assumption to map the problem $\left(A, \delta ; u_{1}, u_{2}\right)$ into a pair $(S, d)$, where $S \subseteq \mathbb{R}^{2}$ and $d \in S$ are respectively the (convex hull of the) image of $A$ and the image of $\bar{\delta}$. After the mapping from $\left(A, \delta ; u_{1}, u_{2}\right)$ to $(S, d)$ is established, Nash's axioms concern sets or points in $\mathbb{R}^{2}$.

Mapping the original bargaining problem to $\mathbb{R}^{2}$ makes for a simple and elegant axiomatization, but Nash's approach forcibly casts $(S, d)$ into a utility-based language; see Rubinstein, Safra, and Thomson (1992). In the next section, we cast an analogous mapping in a probability-based language (LiCalzi, 1999) and axiomatize a procedural solution that nests the Nash solution. Our approach is consistent with, but does not require, the assumption that bargainers have expected utility preferences.

\section{MEDIATOR-ASSISTED NASH BARGAINING}

Let $\left(A, \delta\right.$; $\succsim_{1}, \succsim_{2}$ ) be a bargaining problem with ordinal preferences. Consider a third party - a mediator-who is hired by the two bargainers to recommend an alternative over which they can reach an agreement. The mediator can choose any alternative from $A$, but cannot impose it. After she irrevocably selects a proposal $a$, each bargainer decidesindividually and simultaneously-whether to accept or refuse it. The mediation is successful if both accept the mediator's proposal; otherwise, the bargainers obtain the default outcome $\delta$.

\footnotetext{
${ }^{2}$ Nash (1950) offered no justification for this modeling choice. Bleichrodt, Li, Moscati, and Wakker (2016) suggested why he might have found it so compelling to eschew a discussion.
} 
The mediator's goal is to maximize the probability of a successful mediation in a specific bargaining problem. We expect her to acquaint herself with all relevant information, including bargainers' claims, prevailing social norms, customary or legal precedents, and other contextual elements that bear relevance. Eventually, she confronts her (subjective) uncertainty about what each bargainer $i$ is willing to accept and for any alternative $a$ she turns to the question: "how likely is bargainer i to accept this proposal?"

Having reached her best understanding of the bargaining problem under consideration, the mediator assesses the probability $P_{i}(a)$ that the alternative $a$ will be accepted by bargainer $i$. Let $P_{i}: A \rightarrow[0,1]$ denote the mediator's assessment for bargainer $i=1,2$. This assessment might be generated by a model of $i$ 's behavior as in the example from the Introduction, but this is not a requirement. We assume only that the two mediator's assessments are continuous and satisfy two minimal properties of consistency with her knowledge of the bargaining problem.

\section{C.1-Monotonicity: $P_{i}(a)$ represents $\succsim_{i}$ on $A$.}

This states that the mediator ranks the acceptance probabilities for each bargainer $i$ consistently with $i$ 's ordinal preferences over alternatives; that is, $i$ likes $a$ better than $a^{\prime}$ if and only if the mediator believes that $i$ is more likely to accept $a$ than $a^{\prime}$.

\section{C.2-Bargainers' rationality: $P_{i}(\delta)=0$ and $P_{i}\left(M_{i}\right)=1$.}

The first part states that the mediator believes that $i$ would refuse the default outcome for sure, because he could secure it without going through the hassle of bargaining. The second part states that she believes that $i$ would accept for sure his best choice $M_{i}$ from the set $A^{*}$ : a rational bargainer $i$ knows that he cannot obtain more than $M_{i}$, because $j$ would veto it. If he were not willing to accept his best choice $M_{i}$, he would have refused to enter (or would have walked away from) the bargaining situation. Thus, conditional on $i$ 's status as a bargaining party, he must be willing to accept $M_{i}$.

Section 5 axiomatizes a benchmarking procedure by which the mediator can construct a consistent assessment $P_{i}$ against a standard of reference. Theorem 7 provides sufficient conditions for the existence of two continuous assessments $P_{1}$ and $P_{2}$ that satisfy C.1 and C.2. For expositional purposes, the current and the next section assume that the pair $\left(P_{1}, P_{2}\right)$ of mediator's assessments is given.

The pair $\left(P_{1}, P_{2}\right)$ maps a bargaining problem with ordinal preferences $\left(A, \delta ; \succsim_{1}, \succsim_{2}\right)$ into a subset $B \subseteq[0,1]^{2}$, where each element of $B$ is a pair of acceptance probabilities $\left(p_{1}, p_{2}\right)$; see Figure 1 . The default outcome $\delta$ is mapped to the point $\left(P_{1}(\delta), P_{2}(\delta)\right)=$ $(0,0)$; thus $B$ contains the origin. The best choices $M_{1}$ and $M_{2}$ are mapped to two points $\left(1, p_{2}\right)$ and $\left(p_{1}, 1\right)$, with $p_{2}=P_{2}\left(M_{1}\right)$ and $p_{1}=P_{1}\left(M_{2}\right)$ : both the intersections of $B$ with the line $p_{1}=1$ and with the line $p_{2}=1$ are not empty. Finally, the image of the (compact) Pareto optimal set $A^{*}$ maps to a (compact) Pareto frontier $B^{*}$ in $[0,1]$, because $P_{1}$ and $P_{2}$ are continuous.

In short, the mediator's assessments map the bargaining problem with ordinal preferences $\left(A, \delta ; \succsim_{1}, \succsim_{2}\right)$ to a subset $B \subseteq[0,1]^{2}$ of pairs of acceptance probabilities $\left(p_{1}, p_{2}\right)$, which includes the origin, intersects $p_{1}=1$ and $p_{2}=1$, and has a compact Pareto frontier $B^{*}$.

The analogy with Nash's reformulation of a bargaining problem with EU-preferences as a pair $(S, d)$ should be apparent. The Nash construction uses the mapping $\left(u_{1}, u_{2}\right)$ associated with the two bargainers' utility functions and abstracts away the particulars except for 


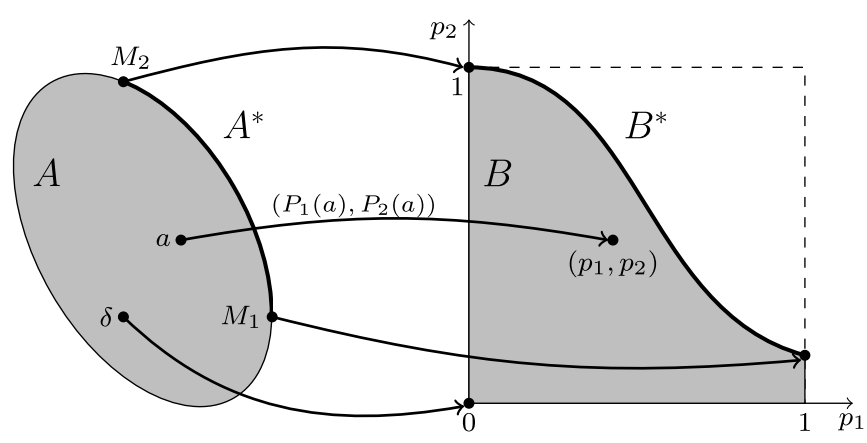

FIGURE 1.-Mapping alternatives to acceptance probabilities.

their risk attitude. ${ }^{3}$ Our construction uses the mapping $\left(P_{1}, P_{2}\right)$ associated with the two mediator's assessments and abstracts away the particulars except for the individual acceptance probabilities. The Nash solution concerns bargaining problems with expected utility preferences. Our solution concerns bargaining problems with mediator's assessments. We discuss in Section 6 how more deeply the analogy runs for unassisted bargaining. The rest of this section provides a behavioral characterization for a procedural solution on $A$, rationalized by the ranking associated with the product $P_{1}(a) \cdot P_{2}(a)$.

\section{A Behavioral Characterization}

We assume that the mediator has a ranking over all pairs of acceptance probabilities in $[0,1]^{2}$, consistent with her goal to maximize the probability of a successful mediation. Intuitively, one may imagine that she aggregates her (individual) probability assessments $P_{1}$ and $P_{2}$ into a single (joint) assessment for the probability of a successful mediation. The equivalent of the Nash solution is recovered when the two mediator's assessments are stochastically independent: then the probability that an alternative $a$ is accepted by both bargainers is the product of $P_{1}(a)$ and $P_{2}(a)$.

Denote by $\mathbf{p}=\left(p_{1}, p_{2}\right)$ an element in $[0,1]^{2}$. We view $[0,1]^{2}$ as a mixture space for the $\oplus$ operation, under the standard interpretation where $\alpha \mathbf{p} \oplus(1-\alpha) \mathbf{q}$ is a lottery that delivers $\mathbf{p}$ in $[0,1]^{2}$ with probability $\alpha$ in $[0,1]$ and $\mathbf{q}$ in $[0,1]^{2}$ with the complementary probability $1-\alpha$; see Herstein and Milnor (1953). Moreover, let $\mathbf{p} \vee \mathbf{q}=$ $\left(\max \left(p_{1}, q_{1}\right), \max \left(p_{2}, q_{2}\right)\right)$ and $\mathbf{p} \wedge \mathbf{q}=\left(\min \left(p_{1}, q_{1}\right), \min \left(p_{2}, q_{2}\right)\right)$.

We assume that the mediator's ranking $\succeq$ over $[0,1]^{2}$ satisfies four properties.

A.1-Regularity: $\succeq$ is a complete preorder, continuous and mixture independent.

This implies that there exists a real-valued function $V:[0,1]^{2} \rightarrow[0,1]$, unique up to increasing affine transformations, that represents the mediator's ranking $\succeq$ and is linear with respect to $\oplus$; that is, $V(\alpha \mathbf{p} \oplus(1-\alpha) \mathbf{q})=\alpha V(\mathbf{p})+(1-\alpha) V(\mathbf{q})$, for any $\alpha$ in $[0,1]$ and any $\mathbf{p}, \mathbf{q}$ in $[0,1]^{2}$. See Theorem 8.4 in Fishburn (1970). Nash (1950, p. 157) took for granted a similar assumption under EU-preferences: he defined a "two-person anticipation as a combination of two one-person anticipations" and stated that the two-person anticipation "will have the same linearity property."

\footnotetext{
${ }^{3}$ This is an implicit assumption of invariance to isomorphic transformations, formalized in Valenciano and Zarzuelo (1997).
} 


\section{A.2-Non-triviality: $(1,1) \succ(0,0)$.}

This states that the mediator ranks a proposal accepted for sure by both bargainers strictly above a proposal refused for sure by both bargainers. Its purpose is to rule out the trivial case where the mediator is always indifferent.

\section{A.3-Disagreement indifference: For any $p, q$ in $[0,1],(p, 0) \sim(0, q)$.}

This is named after Assumption DI in Border and Segal (1997), who studied a preference relation over utility-based solutions. In our framework, this property states that the mediator is indifferent about which bargainer refuses a proposal for sure. Intuitively, the identity of the refuser is irrelevant.

\section{A.4-Consistency: For any $p, q \in[0,1]$,}

$$
p(1, q) \oplus(1-p)(0, q) \sim(p, q) \quad \text { and } \quad p(q, 1) \oplus(1-p)(q, 0) \sim(q, p)
$$

This is a separability property. Suppose that each bargainer $i$ is known to accept a proposal with probability $q$. Then the mediator is indifferent between proposing an alternative that bargainer $j=3-i$ accepts with probability $p$, or facing a lottery where bargainer $j$ accepts with probability $p$ and rejects with probability $1-p$.

Our first result characterizes when the mediator's ranking aggregates pairs of acceptance probabilities using the product rule.

THEOREM 1: The ranking $\succeq$ on $[0,1]^{2}$ satisfies A.1-A.2-A.3-A.4 if and only if it is represented by the product $p_{1} \cdot p_{2}$.

This result uncovers an appealing interpretation for the product operator underlying the Nash solution. Given her probability assessments $P_{1}, P_{2}$ on $A$, the mediator's ranking over pairs of acceptance probabilities in $[0,1]^{2}$ induces a preference relation over alternatives in $A$ represented by the function $V(a)=P_{1}(a) \cdot P_{2}(a)$. Thus, she recommends an alternative $a$ that maximizes the product $P_{1}(a) \cdot P_{2}(a)$. If the bargaining problem is framed with respect to acceptance probabilities (instead of utilities), the product operator corresponds to the assumption that the individual acceptance probabilities are stochastically independent. ${ }^{4}$

Our result is a behavioral characterization. However, it also fits the normative view expounded in Border and Segal (1997, p. 5), who interpreted "axioms as characteristics of the arbitrator that both bargainers can accept." Similarly, it is consistent with the practitioners' view as argued by Subramanian (2010, p. 109): “the implications for negotiation strategy change dramatically when we move away from the assumption that dealmakers will accept deals that are just better than their [default outcome] to the more realistic and nuanced assumption that the likelihood the other side will say yes increases with the incentives to do so."

\footnotetext{
${ }^{4}$ For expositional simplicity, we assume two bargainers. An extension of Theorem 1 to the case of $n \geq 2$ bargainers is given in Appendix A.5.
} 


\section{MEDIATOR-ASSISTED BARGAINING}

\section{An Example}

Theorem 1 axiomatizes the case where the mediator postulates that the individual acceptance probabilities are stochastically independent. For example, let us return to the case where the mediator is to divide a homogeneous cake of unit size by offering portions of size $x$ to $i=1$ and of size $1-x$ to $i=2$. If the mediator believes that the bargainers' targets have i.i.d. uniform distributions on $[0,1]$, she maximizes the probability of reaching an agreement by solving

$$
\max _{x \in[0,1]} F_{1}(x) \cdot F_{2}(1-x)=x(1-x)
$$

and recommends the division where 1 receives $x^{*}=\frac{1}{2}$.

The mediator might want to disallow the premise of stochastic independence. For example, imagine that the mediator believes that the joint distribution for the bargainers' targets is uniform, but is convinced that 1 has a more ambitious target than 2 and so $T_{1} \geq T_{2}$. Using the theory of order statistics, the marginal cumulative distributions for the bargainers' targets are $F_{1}(x)=x^{2}$ and $F_{2}(y)=2 y-y^{2}$ for $x, y \in[0,1]$.

Consistent with the information that $T_{1} \geq T_{2}$, the mediator conjectures that 1 should be offered a larger portion than 2 , that is, $x^{*} \geq \frac{1}{2}$. If she incorrectly maintains the assumption of stochastic independence and uses the Nash product, she solves

$$
\max _{x \in[0,1]} F_{1}(x) F_{2}(1-x)=x^{2}\left[2(1-x)-(1-x)^{2}\right]=x^{2}\left(1-x^{2}\right)
$$

and recommends the division where 1 receives $x^{N}=\frac{\sqrt{2}}{2}$. However, since the joint probability of acceptance under a division $(x, 1-x)$ with $x \geq \frac{1}{2}$ is $F(x, 1-x)=(3 x-1)(1-x)$, the mediator should maximize this expression and propose instead $x^{*}=\frac{2}{3}<\frac{\sqrt{2}}{2}=x^{N}$. Bargainer 1 should be offered a larger portion than bargainer 2, but less than the Nash solution would recommend.

This section generalizes Theorem 1 and provides a representation result for any dependence structure between individual acceptance probabilities.

\section{The General Characterization}

We assume that the mediator's ranking satisfies five properties. (Appendix A.2 shows that, under the first one, the other four properties are logically independent.) The first three properties A.1-A.2-A.3 have been introduced for mediator-assisted Nash bargaining and need not be stated again. We present only the two new ones.

A. $4^{w}$-Weak consistency: For any $p$ in $[0,1]$,

$$
p(1,1) \oplus(1-p)(0,1) \sim(p, 1) \quad \text { and } \quad p(1,1) \oplus(1-p)(1,0) \sim(1, p) .
$$

This is a weakening of A.4 (Consistency) in Section 3 and states the following. Assume that bargainer $i=1,2$ is believed to accept for sure. Then a lottery where the other party $j=3-i$ accepts with probability $p$ and refuses with probability $(1-p)$ is ranked on a par with a proposal that $j$ accepts with probability $p$. Intuitively, the first lottery has an "objective" probability $p$ of success, while the second proposal has a "subjective" probability with the same value $p$. The mediator is indifferent over these two modalities. 
A. $5^{w}$-Weak complementarity: For any $\mathbf{p}, \mathbf{q}$ in $[0,1]^{2}$,

$$
(1 / 2)(\mathbf{p} \vee \mathbf{q}) \oplus(1 / 2)(\mathbf{p} \wedge \mathbf{q}) \succeq(1 / 2) \mathbf{p} \oplus(1 / 2) \mathbf{q} .
$$

This is an analog of Axiom S in Francetich (2013). If $\left(p_{1}-q_{1}\right)\left(p_{2}-q_{2}\right) \geq 0$, the lefthand side (LHS) and the right-hand side (RHS) are the same and (4.1) holds trivially. Assume $p_{1}>q_{1}$ and $p_{2}<q_{2}$. Then $\mathbf{p} \vee \mathbf{q}=\left(p_{1}, q_{2}\right)$ (respectively, $\left.\mathbf{p} \wedge \mathbf{q}=\left(q_{1}, p_{2}\right)\right)$ rearranges the probabilities in $\mathbf{p}$ and $\mathbf{q}$ and matches the higher (lower) values. Weak complementarity states that the mediator never ranks a fifty-fifty lottery over matched pairs strictly below a fifty-fifty lottery over unmatched pairs. Intuitively, the individual acceptance probabilities are (weakly) complementary with respect to the probability of joint acceptance.

The motivation for A. $5^{w}$ is the following. By C.1 (Monotonicity), $p_{i}=P_{i}(a)$ represents $i$ 's ordinal preferences over $A$. Let $a_{p}$ denote an alternative such that $\mathbf{p}=$ $\left(P_{1}\left(a_{p}\right), P_{2}\left(a_{p}\right)\right)$, with analogous notation for the alternatives $a_{q}, a_{\vee}, a_{\wedge}$. If $p_{1}>q_{1}$ and $p_{2}<q_{2}$, the first agent has preferences $a_{\vee} \sim_{1} a_{p} \succ_{1} a_{q} \sim_{1} a_{\wedge}$ and we say that he has high value for $a_{\vee}$ and $a_{p}$ and low value for $a_{q}$ and $a_{\wedge}$. Similarly, the second agent has high value for $a_{\vee}$ and $a_{q}$ and low value for $a_{p}$ and $a_{\wedge}$. From the viewpoint of either bargainer, the equal-chance lotteries in the LHS and in the RHS of (4.1) deliver either a high or a low value, and thus might be considered indifferent. But the mediator is concerned about the approval of both bargainers: she realizes that the LHS leads to high or low values for both bargainers, whereas the RHS delivers high value for one and low value for the other. Because one disgruntled bargainer (with low value) suffices to put successful bargaining at risk, she ranks the LHS weakly above the RHS.

The next result gives a behavioral characterization for the mediator's ranking. The representation takes as input the two bargainers' individual acceptance probabilities and delivers as output the probability of successful bargaining, via a unique copula function. Appendix A.1 recalls a few notions about copulas.

THEOREM 2: The ranking $\succeq$ on $[0,1]^{2}$ satisfies A.1-A.2-A.3-A. $4^{w}-\mathrm{A} .5^{w}$ if and only if there exists a unique copula $C:[0,1]^{2} \rightarrow[0,1]$ that represents $\succeq$, in the sense that

$$
\mathbf{p} \succeq \mathbf{q} \text { if and only if } C(\mathbf{p}) \geq C(\mathbf{q}) .
$$

This result has a straightforward interpretation. A pair $\left(p_{1}, p_{2}\right)$ of individual acceptance probabilities represents the mediator's beliefs. If her ranking satisfies A.1-A.2-A.3A. $4^{w}-\mathrm{A} .5^{w}$, there exists a (unique) copula $C\left(p_{1}, p_{2}\right)$ aggregating $p_{1}$ and $p_{2}$. The mediator ranks an alternative $a$ mapping to a pair of acceptance probabilities $\left(P_{1}(a), P_{2}(a)\right)=$ $\left(p_{1}, p_{2}\right)$ in $B$ by its probability of joint acceptance $C\left(p_{1}, p_{2}\right)$. For instance, consider the example opening this section: a proposal $(x, 1-x)$ has individual acceptance probabilities $p_{1}=F_{1}(x)=x^{2}$ and $p_{2}=F_{2}(1-x)=1-x^{2}$, with joint distribution $F(x, 1-x)=$ $(3 x-1)(1-x)$. Then the copula delivering the probability of joint acceptance is

$$
C\left(p_{1}, p_{2}\right)= \begin{cases}\left(2 \sqrt{p_{1}}-1+\sqrt{1-p_{2}}\right)\left(1-\sqrt{1-p_{2}}\right) & \text { if } 0 \leq 1-\sqrt{1-p_{2}} \leq \sqrt{p_{1}} \leq 1, \\ p_{1} & \text { otherwise }\end{cases}
$$

Some technical comments are in order. First, Theorem 2 characterizes a ranking $\succeq$ that has a maximal element (and thus a procedural solution exists), because any copula is Lipschitz continuous and $B^{*}$ is compact; note that $B$ is not required to be convex or 
even connected. Second, the procedural solution may not be unique: in general, the set of maximal elements is an equivalence class of pairs of individual acceptance probabilities. Third, the theorem puts no restriction on the dependence structure between $P_{1}$ and $P_{2}$ because the copula is arbitrary. Fourth, the copula is linear with respect to $\oplus$, in the sense that $C(\alpha \mathbf{p} \oplus(1-\alpha) \mathbf{q})=\alpha C(\mathbf{p})+(1-\alpha) C(\mathbf{q})$; the mediator evaluates the probability of success for a lottery over $\mathbf{p}$ and $\mathbf{q}$ by assessing first their respective probabilities of success $C(\mathbf{p})$ and $C(\mathbf{q})$, and then mixing those with the same "objective" weights of the lottery.

There are stronger versions of Theorem 2 . We present two of them, concerning respectively the case when (a) the mediator's ranking $\succeq$ respects the (strong) Pareto ordering; or $(b) \succeq$ satisfies an elementary notion of fairness. These two properties are independent and may hold concurrently. Proofs are omitted, because they are trivial modifications of the proof for Theorem 2.

Because any copula $C$ is (weakly) increasing in each argument, the mediator's ranking $\succeq$ in Theorem 2 satisfies the (weak) Pareto ordering: if $p_{1} \geq q_{1}$ and $p_{2} \geq q_{2}$, then $\left(p_{1}, p_{2}\right) \succeq\left(q_{1}, q_{2}\right)$. But it might violate the (strong) Pareto ordering for which $\left(p_{1}-q_{1}\right)\left(p_{2}-q_{2}\right)>0$ implies $\left(p_{1}, p_{2}\right) \succ\left(q_{1}, q_{2}\right)$. A mild strengthening of A. $5^{w}$ rules out this possibility. Let $\mathbf{p} \bowtie \mathbf{q}$ denote that $\mathbf{p}$ and $\mathbf{q}$ are not comparable in the component-wise ordering; that is, $\left(p_{1}-q_{1}\right)\left(p_{2}-q_{2}\right)<0$.

A.5-Complementarity: For any $\mathbf{p} \bowtie \mathbf{q}$ in $[0,1]^{2}$,

$$
(1 / 2)(\mathbf{p} \vee \mathbf{q}) \oplus(1 / 2)(\mathbf{p} \wedge \mathbf{q}) \succ(1 / 2) \mathbf{p} \oplus(1 / 2) \mathbf{q}
$$

In combination with A.1-A.2-A.3-A. $4^{w}$, this property rules out "thick" indifference curves and implies that $\succeq$ is consistent with the (strong) Pareto ordering. This follows from the next result, because any strictly supermodular copula is strictly increasing in each argument.

THEOREM 3: The ranking $\succeq$ satisfies A.1-A.2-A.3-A. $4^{w}-\mathrm{A} .5$ if and only if it is represented by a unique strictly supermodular copula $C:[0,1]^{2} \rightarrow[0,1]$.

The second version incorporates the requirement that the mediator's ranking $\succeq$ should not be affected by the bargainers' identity.

A.6-Anonymity: For any $p, q$ in $[0,1],(p, q) \sim(q, p)$.

This states that the mediator's ranking for any pair $(p, q)$ of individual acceptance probabilities is invariant to their permutation, and hence is anonymous. The following result is immediate.

THEOREM 4: The ranking $\succeq$ satisfies A.1-A.2-A.3-A. $4^{w}-\mathrm{A} .5^{w}-\mathrm{A} .6$ if and only if it is represented by a unique symmetric copula $C:[0,1]^{2} \rightarrow[0,1]$.

Theorem 2 and its two variants characterize the mediator's ranking as the aggregation (via a suitable copula) of two individual acceptance probabilities into a joint probability of successful bargaining. Their generality leaves unspecified the dependence structure modeled by the copula. Our next results specialize the dependence structure and recover characterizations for the analogs of two other well-known bargaining solutions: egalitarian and (relative) utilitarian. Whereas the Nash solution presumes stochastic independence, these two solutions correspond to the extreme cases of maximal positive dependence and maximal negative dependence, respectively. 


\section{Special Cases}

\section{The Egalitarian Solution}

In the utility-based language consistent with Nash's approach, the egalitarian solution (Kalai, 1977) recommends the point on the Pareto frontier at which utility gains from the disagreement point $d$ are equal. Given a bargaining problem with EU-preferences, the egalitarian solution is procedural because it is rationalized by the ranking associated with the function $\min \left\{\left(u_{1}-d_{1}\right),\left(u_{2}-d_{2}\right)\right\}$.

In a probability-based language, the egalitarian solution for a bargaining problem with ordinal preferences arises when the mediator's ranking $\succeq$ aggregates the two bargainers' acceptance probabilities through the copula $M(p, q)=\min (p, q)$. This copula obtains under the following property.

\section{A.7-Meet indifference: For any $p, q$ in $[0,1],(p, p \wedge q) \sim(p \wedge q, q)$.}

This is an analog of Meet preservation in Voorneveld (2014). It states that the ranking is indifferent when pairs of acceptance probabilities have the same minimum value. Intuitively, the evaluation of a pair $(p, q)$ is consistent with prioritizing the smallest value between $p$ and $q$. Clearly, A.7 implies A.3 (Disagreement indifference) and A.6 (Anonymity). Lemma A.3 in the Appendix shows that A.1-A.2-A.4 $4^{w}-\mathrm{A} .7$ imply A.5 ${ }^{w}$. Then the following characterization of the egalitarian copula is immediate.

THEOREM 5: The ranking $\succeq$ satisfies A.1-A.2-A. $4^{w}-\mathrm{A} .7$ if and only if it is represented by the copula $M(p, q)=\min (p, q)$.

The copula $M(p, q)=\min (p, q)$ is known as the Fréchet upper bound, associated with the strongest possible positive dependence between two marginal distributions. The egalitarian solution recommends to maximize the probability of successful bargaining under the premise that the mediator's assessments are maximally positively dependent. Intuitively, she believes that bargainers' propensities to accept a deal are perfect complements with respect to the probability of successful bargaining.

\section{The Utilitarian Solution}

There exist alternative formulations of the utilitarian solution for the Nash model. They share the general principle that the solution recommends an alternative that maximizes the sum of utilities (or utility increments over the disagreement point). We follow Arrow (1951) and consider relative utilitarianism, based on the sum of cardinal utility functions with range normalized to the interval [0,1]; see Dhillon and Mertens (1999). When using a probability-based language, the normalization is a natural step before mapping the utilitarian precept into the recommendation of maximizing the sum of individual acceptance probabilities.

We show that this recommendation is consistent with maximizing the probability of successful bargaining, when the mediator's assessments are maximally negatively dependent. Consider the following property.

\section{A.8-Average indifference: For any $p, q$ in $[0,1],(p, q) \sim\left(\frac{p+q}{2}, \frac{p+q}{2}\right)$.}

This states that the mediator's ranking does not change if the probability assessments on the segment between $(p, q)$ and $\left(\frac{p+q}{2}, \frac{p+q}{2}\right)$ make the increase of one term exactly compensate the diminution of the other. Intuitively, the mediator believes that the bargainers' propensities to accept a deal are perfect substitutes with respect to the probability of 
successful bargaining. Lemma A.4 in the Appendix shows that A.1-A.2-A. $4^{w}-\mathrm{A} .8$ imply A. $5^{w}$. The following characterization of the utilitarian copula holds.

THEOREM 6: The ranking $\succeq$ satisfies A.1-A.2-A.3-A. $4^{w}-\mathrm{A} .8$ if and only if it is represented by the copula $W(p, q)=\max (p+q-1,0)$.

The copula $W(p, q)=\max (p+q-1,0)$ is known as the Fréchet lower bound, associated with the strongest possibile negative dependence between two marginal distributions. Therefore, this procedural solution recommends to maximize the probability of successful bargaining under the premise that the mediator's assessments are maximally negatively dependent.

The copula $W(p, q)$ is strongly Pareto increasing on the triangle above the diagonal from $(0,1)$ to $(1,0)$, but is zero on the rest of its domain. Hence, the mediator's ranking $\succeq$ characterized in Theorem 6 is indifferent over all pairs below the diagonal, because any feasible proposal mapping to any such pair will be refused and thus it is as good as the default outcome.

\section{Another Class of Solutions}

The literature has other bargaining solutions amenable to our copula-based approach. For example, Blackorby, Bossert, and Donaldson (1994) defined a bargaining solution as the (set of) maximizers for a generalized Gini ordering corresponding to distinct levels of inequality aversion, and represented by a quasi-concave, increasing function that is linear on the rank-ordered subsets of $[0,1]^{2}$. In particular, for $0 \leq \alpha \leq 1 / 2$, let $\Delta_{1} \subseteq[0,1]^{2}$ be the triangle with vertices at $(\alpha, \alpha),(0,1)$, and $(1,1)$ and let $\bar{\Delta}_{2} \subseteq[0,1]^{2}$ be the triangle with vertices at $(\alpha, \alpha),(1,0)$, and $(1,1)$. Then the family of symmetric copulas

$$
C_{\alpha, \alpha}(p, q)= \begin{cases}p-\frac{\alpha}{1 \frac{\alpha}{\alpha}}(1-q) & \text { if }(p, q) \in \Delta_{1} \\ q-\frac{\alpha}{1-\alpha}(1-p) & \text { if }(p, q) \in \Delta_{2} \\ 0 & \text { otherwise }\end{cases}
$$

defines a continuum of (affine) generalized Gini orderings. Blackorby, Bossert, and Donaldson (1994) interpreted $\alpha$ as an index of inequality aversion, whereas our approach views it as an index of stochastic dependence. This family of symmetric copulas includes the egalitarian solution for $\alpha=0$ and the utilitarian solution for $\alpha=1 / 2$. If we drop the analog of A.6 (Anonymity) assumed in Blackorby, Bossert, and Donaldson (1994), this family may be embedded in a more general class of asymmetric two-parameter copulas $C_{\alpha, \beta}$; see Nelsen (2006, Exercise 3.8).

\section{Uniqueness}

A copula-based procedural solution may not be unique, because our approach imposes no convexity assumptions on either $A$ or its image in $[0,1]^{2}$ via the $P_{i}$ 's mappings. Nash (1950, p. 159) stated bluntly: "Convexity makes [the solution] unique." We concur with Blackorby, Bossert, and Donaldson (1994, p. 1162) who argued that the generalized Gini solutions "are multi-valued solutions (unless attention is restricted to strictly convex problems). [...] relaxing this assumption enlarges the class of solutions considerably. Hence, single-valuedness is not merely an assumption of convenience but, rather, an assumption 
of substance." One can recover uniqueness for copula-based solutions by adding adequate richness assumptions on the domain or on the bargainers' preferences. When the solution is not unique, there is a set of alternatives for which the probability of successful bargaining is the same. By analogy with social choice correspondences, Blackorby, Bossert, and Donaldson (1994) suggested that the mediator might issue her final recommendation through a random selection.

\section{BENCHMARKING AND EU-PREFERENCES}

\section{The Benchmarking Procedure}

Given a bargaining problem $\left(A, \delta\right.$; $\left.\succsim_{1}, \succsim_{2}\right)$ with ordinal preferences, its solution is an alternative $a$ in $A$. As described in Figure 1, and consistently with the Nash model, our approach maps the original problem to a subset $B \subseteq[0,1]^{2}$ of acceptance probabilities, finds a solution $\mathbf{p}^{*}$ in $B$, and then maps it back to some $a^{*}$ in $A$. The path to a procedural solution has three stages: (1) reduction of the problem $\left(A, \delta ; \succsim_{1}, \succsim_{2}\right)$ to a simpler representation $B$; (2) axiomatization of the ranking to select $\mathbf{p}^{*}$; (3) retrieval of the corresponding alternative from $A$.

Until now, we have focused on the second stage, that is, the axiomatization. This section is concerned with the first stage and formalizes a procedure to construct the probability assessments that map ( $\left.A, \delta ; \succsim_{1}, \succsim_{2}\right)$ into $B$.

Let $\left(A, \delta\right.$; $\left.\succsim_{1}, \succsim_{2}\right)$ be a bargaining problem with ordinal preferences. We axiomatize a benchmarking procedure by which an agent $k \neq i$ (e.g., the mediator) formulates beliefs consistent with bargainer $i$ 's ordinal preferences, using a standard of reference to calibrate her assessment $P_{k(i)}(a)$ for the probability that $i$ accepts $a$ as bargaining outcome. This procedure generates a unique assessment for the acceptance probabilities of $i$, as perceived by agent $k$.

The standard of reference is the set $\mathcal{L}_{i}$ of simple lotteries over $\left\{M_{i}, \delta\right\}$. A simple lottery $L_{p}=p M_{i} \oplus(1-p) \delta$ in $\mathcal{L}_{i}$ yields $M_{i}$ with probability $p$ in $[0,1]$ and $\delta$ with probability $1-p$. Suppose that $k$ uses $L_{p}$ to select a recommendation for $i$ : with probability $p$, she proposes $M_{i}$ and $i$ accepts, and with probability $1-p$, she proposes $\delta$ and $i$ refuses. Let $\hat{P}_{k(i)}: \mathcal{L}_{i} \rightarrow[0,1]$ be $k$ 's assessment for a lottery $L_{p}$; we assume $\hat{P}_{k(i)}\left(L_{p}\right)=p$ for any $p{ }^{5}$ because the outcome of $L_{p}$ is accepted with (objective) probability $p$.

The benchmarking procedure extends $\hat{P}_{k(i)}$ on $\mathcal{L}_{i}$ to an assessment $P_{k(i)}$ on $A \cup \mathcal{L}_{i}$ by comparing each alternative $a$ against the lotteries in $\mathcal{L}_{i}$ : if agent $k$ feels that $a$ is as likely to be accepted by $i$ as $L_{p}$, she sets $P_{k(i)}(a)=p$.

Formally speaking, let $\succsim_{i}$ be the preference relation of bargainer $i$ on the set of alternatives $A$ and let $\succeq_{k(i)}$ be a preorder on $A \cup \mathcal{L}_{i}$. We interpret an element in $A \cup \mathcal{L}_{i}$ as a proposal to bargainer $i$ : this can be an alternative $a$ or (unbeknown to the bargainer) a Chance decision between $M_{i}$ and $\delta$. The preorder $\succeq_{k(i)}$ represents agent $k$ 's ranking over such proposals, where $x \succeq_{k(i)} y$ if and only if agent $k$ believes that bargainer $i$ is not less likely to accept $x$ than $y$.

The two consistency requirements C. 1 and C. 2 for $P_{i}$ from Section 3 correspond to two conditions on the agent's ranking $\succeq_{k(i)}$ :

B.1-Monotonicity: The preorder $\succeq_{k(i)}$ represents $\succsim_{i}$ on $A$.

\footnotetext{
${ }^{5}$ This is the natural choice, but the construction proceeds essentially unchanged if one replaces $p$ with any continuous and increasing bijection $g(p)$ from $[0,1]$ onto $[0,1]$.
} 
B.2-Bargainers' rationality: $\delta \sim_{k(i)} L_{0}$ and $M_{i} \sim_{k(i)} L_{1}$.

We impose two additional properties. The first one is that shifting probability from $\delta$ to $M_{i}$ increases $k$ 's confidence that $i$ accepts the proposal.

B.3-Stochastic dominance: If $p>q$, then $L_{p} \succ_{k(i)} L_{q}$.

The last condition is technical. The subset $A$ has the relative topology. The set $\mathcal{L}_{i}$ has the natural topology generated by the metric $d\left(L_{p}, L_{q}\right)=|p-q|$. We endow the set $A \cup \mathcal{L}_{i}$ with the disjoint union topology: a set $\mathcal{O} \subset A \cup \mathcal{L}_{i}$ is open if and only if $\mathcal{O} \cap A$ and $\mathcal{O} \cap \mathcal{L}_{i}$ are open in $A$ and in $\mathcal{L}_{i}$, respectively.

B.4-Continuity: The preorder $\succeq_{k(i)}$ is continuous in the disjoint union topology.

Our next result characterizes the benchmarking procedure.

THEOREM 7: Suppose that the preorder $\succeq_{k(i)}$ satisfies B.2-B.3-B.4. Then the benchmarking procedure uniquely extends the assessment $\hat{P}_{k(i)}$ on $\mathcal{L}_{i}$ to a continuous assessment $P_{k(i)}$ on $A \cup \mathcal{L}_{i}$ with $P_{k(i)}(\delta)=0$ and $P_{k(i)}\left(M_{i}\right)=1$, that represents $\succeq_{k(i)}$. Moreover, the restriction of $P_{k(i)}$ to $A$ is continuous and, if B.1 holds, also represents $\succsim_{i}$.

\section{Expected Utility and Target-Based Preferences}

As it turns out, the commonly known EU-preferences in Nash $(1950,1953)$ are a special case of the benchmarking procedure, where the set of feasible proposals includes all randomizations over the available (physical) alternatives in $A$. Under expected utility, the preferences of bargainer $i$ are defined over the set $\mathcal{L}(A)$ of lotteries on $A$. The choice space is no longer $A$ but $\mathcal{L}(A)$, and an EU-preference is represented by the expected value of a vNM-utility function $u_{i}: A \rightarrow \mathbb{R}$, consistent with $\succsim_{i}$ on $A$ and unique up to increasing affine transformations.

Suppose that an agent $k \neq i$ formulates beliefs $P_{k(i)}$ on $\mathcal{L}(A)$ consistent with $i$ 's preferences on this set. Note that $i$ 's preferences and $k$ 's benchmarking concern $\mathcal{L}(A) \supseteq A \cup \mathcal{L}_{i}$ : the agent $k \neq i$ derives beliefs for $i$ 's probability to accept a (randomized) proposal from the larger set $\mathcal{L}(A)$, but her task is made easier because $k$ knows $i$ 's preferences over all lotteries on $A$. Applying B.1 and B.2 on the domain $\mathcal{L}(A)$, it follows immediately that $k$ sets $P_{k(i)}(\alpha)=E u_{i}(\alpha)$ for any (possibly, degenerate) lottery $\alpha \in \mathcal{L}(A)$, where $u_{i}$ is normalized so that $u_{i}(\delta)=0$ and $u_{i}\left(M_{i}\right)=1$. That is, $k$ 's assessment for the probability that $i$ would accept a (randomized) proposal $\alpha$ equates $i$ 's expected utility. ${ }^{6}$

This observation ties nicely the Nash solution and mediator-assisted Nash bargaining. Suppose that the mediator $k \neq i$ faces a bargaining problem with EU-preferences and knows $i$ 's and $j=(3-i)$ 's preferences on $\mathcal{L}(A)$. If her ranking respects B.1 (Monotonicity) on the domain $\mathcal{L}(A)$ of $i$ 's preferences and B.2 (Bargainers' rationality), then she sets $P_{k(i)}(\alpha)=E u_{i}(\alpha)$; hence, her assessment for $i$ 's acceptance probability of a (randomized)

\footnotetext{
${ }^{6}$ Expected utilities and probabilities may sometimes be swapped. When he claimed the equivalence between Zeuthen's and Nash's theories of bargaining, Harsanyi (1956, p. 149) derived Zeuthen's model by postulating that "each party can estimate correctly the probability that the other party will definitely reject a certain offer." After the equivalence had been acknowledged, Harsanyi (1962, p. 29) subsumed Zeuthen's and Nash's theories presuming that both parties "know [...] each other's attitudes towards risk."
} 
proposal $\alpha$ coincides with its expected utility for bargainer $i$. That is, given a bargaining problem with EU-preferences, Theorem 1 states that the mediator ranks alternatives as if she is maximizing the Nash product of the expected utilities for the two bargainers.

The target-based example from the Introduction is another special case of the benchmarking procedure. Suppose that the mediator $k \neq i$ believes that each bargainer $i$ accepts any proposal that meets his own target, but she knows only the probability distributions for $T_{1}$ and $T_{2}$. Then she would set $P_{k(i)}(a)=P\left(a \succsim_{i} T_{i}\right)$; see Castagnoli and LiCalzi (1996). If the mediator believes that $T_{1}$ and $T_{2}$ are stochastically independent and have continuous distributions, Theorem 1 applies again. And, more generally, Theorem 2 deals with the case when independence does not hold.

\section{BILATERAL BARGAINING}

The Nash bargaining model is concerned with the axiomatization of solution concepts, defined as maps from utility-based representations $(S, d)$ to pairs in $S$. Our probabilitybased approach derives procedural solutions as maximal outcomes under a ranking $\succeq$ on $[0,1]^{2}$, attributed to a mediator. We argue that the ranking $\succeq$ and its properties may be ascribed to bilateral bargaining.

Our perspective is inspired by Border and Segal (1997). Motivated by their variety in the literature, they studied preferences over solution concepts and offered a set of axioms under which the Nash solution is unanimously preferred. Our approach is different because we characterize a ranking over pairs of acceptance probabilities. But they advanced two interpretations that are germane to our discussion.

The first interpretation is that the two bargainers delegate the decision to a third party and "hire an arbitrator to make choices for them" (p. 1). Differently from the mediator, the arbitrator imposes her choice. Then the axioms circumscribe what notion of fairness underlies her ranking. As in social choice, this viewpoint is normative. ${ }^{7}$

The second ancillary interpretation is prescriptive: the bargainers agree to submit the axioms as ex ante guidelines to the arbitrator. These need not represent the bargainers' true preferences over the final choice, but must be consistent with a genuine effort to consent to ex ante mutually acceptable principles. Framing bargaining in a probabilitybased language is consistent with either interpretation.

Finally, imagine two bargainers who formulate ex ante a shared ranking $\succeq$ on $[0,1]^{2}$ under a veil of ignorance: they do not know which alternatives will be available and under which circumstances they will bargain. If they channel the uncertainty about the future into acceptance probabilities, they should agree on a ranking that maximizes the (future) chances of successful bargaining.

The implications are different when we consider the reduction stage that precedes the axiomatization stage in the path to a solution. The reduction stage concerns the modeling of a specific bargaining problem: it should allow for great flexibility. Nash entrusted this burden with the bargainers' vNM-utilities $u_{1}(a)$ and $u_{2}(a)$, focusing on their risk attitudes. Our probability-based approach offers a richer perspective, based on the mediator's (or arbitrator's) beliefs about the individual acceptance probabilities $P_{1}(a)$ and $P_{2}(a)$ for the situation at hand.

There are other cases when $P_{1}(a)$ and $P_{2}(a)$ have a natural interpretation. Consider two negotiators who act on behalf of their principals: they have common knowledge of

\footnotetext{
${ }^{7}$ Kaneko and Nakamura (1979) and Mariotti and Veneziani (2018) characterized social welfare functions based on the Nash product. When the ranking $\succeq$ is a social preference relation, our results characterize social welfare functions based on copulas.
} 
the principals' acceptance probabilities and seek a deal to submit for the principals' approval. For instance, think of two diplomats negotiating a treaty on behalf of their governments. More generally, if there is common knowledge that both bargainers have the same incomplete information about their acceptance probabilities, then they are in agreement on $P_{1}(a)$ and $P_{2}(a)$. Compare with Nash's assumption that there is common knowledge that the bargainers have EU-preferences with given vNM-utility functions.

There is another distinction between the two stages. Reduction is usually carried out ad interim, after a specific problem is presented and before a solution is offered; characterization applies to an abstract space and is discussed ex ante. The reduction stage poses a challenge, first acknowledged in Harsanyi (1962). Given the solution concept downstream, mapping a bargaining problem to a subset of $\mathbb{R}^{2}$ leaves room for manipulation. Mediator-assisted bargaining avoids this issue because the mediator's only interest is that both bargainers agree to her proposal. (Consider what could happen if bribes were allowed.)

The problem becomes relevant in unassisted bargaining, when a third party is not available. Nash shut down the risk of manipulation by imposing that agents' EU-preferences are common knowledge. Our approach is consistent with this strong assumption: if the bargainers' EU-preferences or the distribution of their targets are commonly known, the benchmarking procedure leaves no room for manipulation.

Harsanyi (1962) considered "the more general case where the two parties do not know (and know they do not know) each other's utility functions." He identified two conditions that mitigate the problem. The first one occurs when "in a given society with wellestablished cultural traditions people tend to enter bargaining situations with more or less consistent expectations about each other's utility functions." People from the same culture may use observable features (such as age, social position, education) to reliably assess others' preferences or others' acceptance probabilities. The second condition occurs when consistent expectations are "the result of mutual adjustment [...] during the bargaining process itself."

Another approach that we do not pursue here is the design of strategy-proof mechanisms for eliciting truthful reduction. Miyagawa (2002) took a first step and provided a simple four-stage sequential game that fully implements a reasonably large class of twoperson bargaining solutions in subgame-perfect equilibrium. Compared to our setup, the crucial restriction is that the copula must be quasi-concave. This does not hold in general, but many common examples-including the three copulas characterized aboveare quasi-concave. Therefore, the Nash, egalitarian, and utilitarian solutions are implementable.

\section{COMMENTARY}

This section offers closing comments, organized in two parts. Section 7.1 reviews the ordinal Nash solution by Rubinstein, Safra, and Thomson (1992) and shows that it is consistent with our probability-based approach. Section 7.2 deals with applications, covering testable restrictions, comparative statics, and bargaining power.

\subsection{The Ordinal Nash Solution}

Rubinstein, Safra, and Thomson (1992, p. 1172) put forward a "switch from utility language to alternatives-preference language" for the Nash bargaining model. Based on this language, they introduced the ordinal Nash solution for a class of bargainers' preferences larger than expected utility. 
Specifically, suppose that each bargainer has a preference relation $\succsim_{i}$ over the space $\mathcal{L}(A)$ of lotteries on $A$. An alternative $a^{*}$ from $A$ is an ordinal Nash solution if $p a \oplus(1-$ $p) \delta \succ_{i} a^{*}$ implies $p a^{*} \oplus(1-p) \delta \succsim_{j} a$, for all $p$ in $(0,1]$ and $a$ in $A$. If both agents have EU-preferences over $\mathcal{L}(A)$, the ordinal Nash solution coincides with the standard Nash solution. But their richer language interprets the ordinal Nash solution as an alternative for which no bargainer can raise an admissible objection.

Grant and Kajii (1995) extended this approach to a wider class of preferences over $\mathcal{L}(A)$ and developed a cardinal characterization for the ordinal Nash solution, recovering it as the maximizer of a Nash product. Appendix A.4 provides the details and a mild generalization of their results, but the gist of their argument is the following.

Grant and Kajii (1995) assumed that each bargainer $i=1,2$ has a preference relation $\succsim_{i}$ over the space $\mathcal{L}(A)$. The preference relation $\succsim_{i}$ has a disagreement linear (DL) representation if there exists a continuous function $v_{i}(a)$ (with $\left.v_{i}(\delta)=0\right)$ such that

$$
p a \oplus(1-p) \delta \succsim_{i} q a^{\prime} \oplus(1-q) \delta \quad \text { if and only if } \quad p v_{i}(a) \geq q v_{i}\left(a^{\prime}\right) .
$$

Lemma 1 in Grant and Kajii (1995) characterizes a class of preferences that have a DL representation. These include expected utility and some examples of rank-dependent utility. Lemma 2 shows that $a^{*}$ is an ordinal Nash solution if and only if it maximizes the Nash product $v_{1}(a) \cdot v_{2}(a)$ over the set $A$ of the alternatives.

In our setup, the bargainers have preferences with a DL representation where $v_{i}(a)=$ $P_{i}(a)$. Therefore, their Lemma 2 applies and the Nash copula delivers the same alternatives as the ordinal Nash solution. We do not pursue it here, but one may extend the benchmarking procedure beyond DL representations by using a standard of reference different from $\hat{P}_{k(i)}\left(L_{p}\right)=p$; see Footnote 5 .

\subsection{Modeling Issues}

\section{Testable Restrictions}

We describe a simple test that generates falsifiable predictions for our copula-based approach. For any problem $B$ in $[0,1]^{2}$, a solution selects (at least) a point in $B$. The copula-based approach recommends a solution by maximizing a suitable copula $C$ over $B$. Suppose that $B$ contains a point $\mathbf{p}=\left(p_{1}, p_{2}\right)$ with $p_{1}+p_{2}>1$. The Fréchet lower bound implies $C(\mathbf{p}) \geq p_{1}+p_{2}-1>0$ for any copula $C$. Similarly, given another feasible point $\mathbf{q}=\left(q_{1}, q_{2}\right)$, the Fréchet upper bound implies $C(\mathbf{q}) \leq \min \left(q_{1}, q_{2}\right)$. Therefore, if $\min \left(q_{1}, q_{2}\right)<p_{1}+p_{2}-1$, then $C(\mathbf{p})>C(\mathbf{q})$ : the point $\mathbf{p}$ must be strictly preferred to $\mathbf{q}$, and $\mathbf{q}$ cannot be in the solution for any copula $C$. By picking a point $\mathbf{p}^{*}$ that maximizes $p_{1}+p_{2}-1$ in $B$, we can formulate the following more stringent test.

Proposition 8: Let $\mathbf{p}^{*} \in \arg \max _{B}\left(p_{1}+p_{2}-1\right)$. Define the quadrant

$$
Q:=\left\{\mathbf{q} \in[0,1]^{2}: \min \left(q_{1}, q_{2}\right) \geq p_{1}^{*}+p_{2}^{*}-1\right\} .
$$

Then the solution must belong to $B \cap Q$.

The copula-based approach implies other restrictions. Because a procedural solution is rationalized by the maximization of a copula, it can recover only solutions that satisfy independence of irrelevant alternatives; see Peters and Wakker (1991). In particular, our model cannot yield the Kalai and Smorodinsky (1975) solution (for short, KS solution) 
except in special cases. Formally speaking, this solution violates A.1 as shown by the following simple example. Let $B_{1}$ be the convex hull in $[0,1]^{2}$ of the three points $(0,0)$, $(0,1)$, and $(1,0)$. Then the KS solution uniquely prescribes the point $\mathbf{p}=(1 / 2,1 / 2)$. On the other hand, if $B_{2}$ is the convex hull in $[0,1]^{2}$ of the four points $(0,0),(0,1),(1 / 2,1 / 2)$, and $(1 / 2,0)$, then the KS solution uniquely prescribes the point $\mathbf{q}=(1 / 3,2 / 3)$. As both $\mathbf{p}$ and $\mathbf{q}$ belong to $B_{2} \subset B_{1}$, the first KS solution reveals $\mathbf{p} \succ \mathbf{q}$ while the second one reveals $\mathbf{q} \succ \mathbf{p}$, so A. 1 fails. It is possible to rationalize the KS solution as the outcome of a lexicographic maximization, but we find this approach cumbersome and do not pursue it here.

\section{Comparative Statics}

We make three observations. First, our approach allows for comparative statics based on (partial) orderings for copulas. We consider a simple example. Given two copulas $C_{1}$ and $C_{2}$, the concordance ordering states that $C_{1}$ is more concordant than $C_{2}$ if $C_{1}(\mathbf{p}) \geq$ $C_{2}$ (p) for all $\mathbf{p}$ in $[0,1]^{2}$. Suppose that $C_{1}$ is more concordant than $C_{2}$. Given $B$, let $\mathbf{p}_{i}^{*}$ be a solution under the copula $C_{i}$, for $i=1,2$. Since

$$
C_{1}\left(\mathbf{p}_{1}^{*}\right) \geq C_{1}\left(\mathbf{p}_{2}^{*}\right) \geq C_{2}\left(\mathbf{p}_{2}^{*}\right),
$$

the joint probability of success at the solution is increasing in the concordance ordering. Agents with concordant targets are more likely to strike a deal.

Second, we can reinterpret known results. For example, consider Theorem 1 in Kihlstrom, Roth, and Schmeidler (1981): “The utility which Nash's solution assigns to a player increases as his opponent becomes more risk averse." This result compares the solution when the utility $u_{i}$ of an agent is replaced by a function $v_{i}$ that is an increasing concave transformation of $u_{i}$. Recall from Section 5 that the probability assessment $P_{k(i)}(a)$ may be set equal to the vNM-utility function $u_{i}(a)$ with $u_{i}(\delta)=0$ and $u_{i}\left(M_{i}\right)=1$. Then Theorem 1 in Kihlstrom, Roth, and Schmeidler (1981) states that the Nash copula makes $j=3-i$ better off when the probability assessment $P_{k(i)}$ is replaced by an assessment $Q_{k(i)}$ that is an increasing concave transformation of $P_{k(i)}$. Because probability assessments are normalized to [0,1], this implies $Q_{k(i)}(a) \geq P_{k(i)}(a)$ for any $a$. Hence, the Nash copula makes $j=3-i$ better off if the assessment for $i$ is more optimistic. Under target-based preferences, this occurs when $i$ 's target is less demanding in the sense of first-order dominance. In general, whenever $i$ is believed to be more accommodating, the Nash copula rewards the other bargainer.

The third observation points out to comparative statics beyond the risk attitudes. Consider the division of a homogeneous cake of unit size when both bargainers have targetbased preferences that are unimodal; that is, the distribution function $F_{i}(x)$ for the target $T_{i}$ is S-shaped with an inflection point at its mode $x=m_{i}{ }^{8}$ We are interested in the comparative statics for $x^{*}$ (the portion of the first bargainer) with respect to the two modes $m_{1}, m_{2}$.

As an example, for $m$ in $[0,1)$, consider the family of distributions

$$
F_{i}(x)=\frac{x^{2}}{x^{2}+q\left(m_{i}\right)(1-x)^{2}} \quad \text { for } x \in[0,1]
$$

\footnotetext{
${ }^{8}$ This is more general than assuming that a density function exists and has a maximum at $m_{i}$.
} 
where

$$
q(m)=\frac{m^{2}(3-2 m)}{(1-m)^{2}(1+2 m)}
$$

is a strictly increasing (and hence, injective) convex function from $[0,1)$ onto $\mathbb{R}^{+}$. Each distribution has a unique mode at $m$ and the family is ordered in the sense of first-order dominance with respect to $m$.

Given the bargaining problem

$$
\max F_{1}(x) F_{2}(1-x)=\frac{x^{2}}{x^{2}+q\left(m_{1}\right)(1-x)^{2}} \cdot \frac{(1-x)^{2}}{(1-x)^{2}+q\left(m_{2}\right) x^{2}},
$$

the maximizer $x^{*}\left(m_{1}, m_{2}\right)$ satisfies

$$
\left(\frac{x}{1-x}\right)^{4}=\frac{q\left(m_{1}\right)}{q\left(m_{2}\right)}
$$

and we obtain

$$
x^{*}\left(m_{1}, m_{2}\right)=\frac{\sqrt[4]{q\left(m_{1}\right)}}{\sqrt[4]{q\left(m_{1}\right)}+\sqrt[4]{q\left(m_{2}\right)}} .
$$

Using the properties of $q(m)$, it follows that $x^{*}\left(m_{1}, m_{2}\right)$ is increasing in $m_{1}$ and decreasing in $m_{2}$, ranging onto [0,1] with $x^{*} \geq 1 / 2$ if $m_{1} \geq m_{2}$. As $m_{1}$ increases, the first bargainer becomes more demanding and the Nash copula offers him a larger portion.

This example highlights a connection between unimodal targets and S-shaped utilities; see LiCalzi (1999). Suppose that each agent $i$ has EU-preferences with a vNM-utility function $u_{i}(x)$ and a reference point $m_{i}$ such that he is risk averse over gains $\left(x>m_{i}\right)$ and risk seeking over losses $\left(x<m_{i}\right)$. Then the Nash solution $x^{*}$ for the utility-based model is the same as for the unimodal targets, after replacing $u_{i}(x)$ for $F_{i}(x)$.

Continuing the example, agent $i$ is also loss averse if $m_{i} \geq 1 / 2$. When both bargainers are loss averse, one can show that $x^{*} \leq m_{1}$ and $1-x^{*} \leq m_{2}$. When the reference points for two loss averse bargainers are too high, the Nash copula delivers a solution where each of them receives a portion lower than his reference point: they both end up in their "loss" domain. See Shalev (2002) for some results on bargaining and loss aversion.

\section{Bargaining Power}

The economic literature uses an asymmetric version of the Nash solution as a reduced form for differences in the agents' bargaining power. Given a Nash bargaining problem $(S, d)$, the asymmetric Nash solution obtains as the maximizer of the product $\left(u_{1}-d_{1}\right)^{b_{1}}\left(u_{2}-d_{2}\right)^{b_{2}}$, where $b_{i} \geq 0$ denotes the bargaining power of $i=1,2$. This solution favors the first agent as his relative strength $s=b_{1} /\left(b_{1}+b_{2}\right)$ increases from 0 to 1 . The popularity of the asymmetric Nash solution is due to its technical convenience, without much concern for its foundations or interpretation; see Harsanyi and Selten (1972) for a notable exception.

Our approach allows for asymmetric copulas, but it cannot replicate the asymmetric Nash solution for all $s$. The maximand $N_{s}(p, q)=p^{s} q^{1-s}$ is not a copula because it fails D.2 in Section A.1. One may consider other copulas. For instance, by Theorem 2.1 in Liebscher (2008), for any copula $C$, the expression

$$
C^{s}(p, q)=p^{s} q^{1-s} C\left(p^{1-s}, q^{s}\right)=N_{s}(p, q) \cdot C\left(p^{1-s}, q^{s}\right)
$$


defines an asymmetric copula related to $N_{s}$. However, there are technical limitations. Let $\Delta(C)=\sup _{0 \leq p, q \leq 1}|C(p, q)-C(q, p)|$ be the degree of asymmetry for a function $C$ over the domain $[0,1]^{2}$. It is known that $\Delta(C) \leq 1 / 3$ for any copula $C$ (Nelsen, 2007); and, if $C$ is also quasi-concave, then $\Delta(C) \leq 1 / 5$ (Alvoni and Papini, 2007). Computing the degree of asymmetry for $N_{s}$ yields

$$
\Delta\left(N_{s}\right)=\left|\left(\frac{1-s}{s}\right)^{\frac{1-s}{2 s-1}}-\left(\frac{1-s}{s}\right)^{\frac{s}{2 s-1}}\right| .
$$

This is symmetric around $s=1 / 2$ and increasing in $|s-1 / 2|$; its range goes from $\Delta\left(N_{s}\right)=0$ at $s=1 / 2$ to $\Delta\left(N_{s}\right)=1$ at $s=0$ or $s=1$. In particular, because $\Delta\left(N_{s}\right)>1 / 3$ for $|s-1 / 2|>$ 0.22 , no copula can replicate $N_{s}$ when $s$ is outside of the interval $(.28, .72)$.

Nonetheless, our probability-based approach can recover the asymmetric Nash solution when the bargaining problem concerns a homogeneous cake of unit size and agents have target-based preferences. Suppose that the target $T_{i}$ for $i=1,2$ has a distribution $P\left(x \succsim_{i}\right.$ $\left.T_{i}\right)=x^{b_{i}}$ on the support [0,1]: a greater $b_{i}$ models a more demanding agent $i$, whose target $T_{i}$ is higher in the sense of stochastic dominance. Then maximizing the Nash copula returns the asymmetric Nash solution.

\section{APPENDIX}

\section{A.1. Basics on Bivariate Copulas}

Copulas are functions that link multivariate distributions to their one-dimensional marginal distributions; see Nelsen (2006). They are used to model stochastic dependence. We summarize some basic notions about bivariate copulas.

A (bivariate) copula is a function $C:[0,1]^{2} \rightarrow[0,1]$ that satisfies two properties:

(D.1) for any $p, q$ in $[0,1], C(p, 0)=C(0, q)=0, C(p, 1)=p$, and $C(1, q)=q$;

(D.2) for any $p_{1}>q_{1}$ and $p_{2}>q_{2}$ in $[0,1], C\left(p_{1}, p_{2}\right)+C\left(q_{1}, q_{2}\right) \geq C\left(p_{1}, q_{2}\right)+$ $C\left(q_{1}, p_{2}\right)$.

Property D. 2 is often called supermodularity. The combination of D.1 and D.2 implies that $C(p, q)$ is increasing in each argument; see Nelsen (2006, Lemma 2.1.4). When the weak inequality in D.2 is replaced by a strict one, the copula $C$ is strictly supermodular and strictly increasing in each argument.

A characterization from Sklar (1959) elucidates how the copula connects a bivariate distribution to its univariate marginals.

THEOREM פ:et $(X, Y)$ be a random vector with marginal distributions $F(x)$ and $G(y)$. The following are equivalent:

(i) $H(x, y)$ is the joint distribution function of $(X, Y)$;

(ii) there exists a copula $C(p, q)$ such that $H(x, y)=C[F(x), G(y)]$ for all $x, y$. If $F(x)$ and $G(y)$ are continuous, then $C(p, q)$ is unique. Otherwise, $C(p, q)$ is uniquely defined on the cartesian product $\operatorname{Ran}(F) \times \operatorname{Ran}(G)$ of the ranges of the two marginal distributions. Conversely, if $C(p, q)$ is a copula and $F(x)$ and $G(y)$ are distribution functions, then the function $H(x, y)$ in (ii) is a joint distribution function with marginal distributions $F(x)$ and $G(y)$.

A prominent example of a copula is the product $\Pi(p, q)=p \cdot q$ associated with stochastic independence. Other important examples are $W(p, q)=\max (p+q-1,0)$ and 
$M(p, q)=\min (p, q)$. These two copulas are respectively known as the Fréchet lower and upper bound, because $W(p, q) \leq C(p, q) \leq M(p, q)$, for any copula $C$ and any $(p, q)$ in $[0,1]^{2}$. Intuitively, the copula $M$ describes the strongest possible positive dependence between $X$ and $Y$, given the marginal distributions $F$ and $G$. Similarly, $W$ captures the strongest possible negative dependence.

\section{A.2. Logical Independence of $A .2-A .3-A .4^{w}-A .5^{w}$}

We show that, given A.1, the four axioms A.2-A.3-A. $4^{w}-\mathrm{A} .5^{w}$ in Theorem 2 are logically independent. Recall that A.1 implies that the preference relation $\succeq$ is representable by a real-valued function $V:[0,1]^{2} \rightarrow[0,1]$, unique up to increasing affine transformations and linear with respect to $\oplus$. We provide a counterexample for each property, omitting obvious quantifiers.

\section{A.2 (Non-Triviality)}

Consider $V(p, q)=k$, for some constant $k$ in $[0,1]$. Then A.3 holds because $V(p, 0)=$ $k=V(0, q)$; A. $4^{w}$ holds because $p V(1,1)+(1-p) V(0,1)=k=V(p, 1)$, and similarly for the second relation. And A. $5^{w}$ holds because $(1 / 2) V(\mathbf{p} \vee \mathbf{q})+(1 / 2) V(\mathbf{p} \wedge \mathbf{q})=k=$ $(1 / 2) V(\mathbf{p})+(1 / 2) V(\mathbf{q})$. But A.2 does not hold because $V(1,1)=k=V(0,0)$.

\section{A.3 (Disagreement Indifference)}

Consider $V(p, q)=p$. Then A.2 holds because $V(1,1)=1>0=V(0,0)$; A. $4^{w}$ holds because $p V(1,1)+(1-p) V(0,1)=p=V(p, 1)$, and $p V(1,1)+(1-p) V(1,0)=1=$ $V(1, p)$. And A. $5^{w}$ holds because

$$
\frac{1}{2} V(\mathbf{p} \vee \mathbf{q})+\frac{1}{2} V(\mathbf{p} \wedge \mathbf{q})=\frac{1}{2}\left(p_{1} \vee q_{1}\right)+\frac{1}{2}\left(p_{1} \wedge q_{1}\right)=\frac{1}{2} p_{1}+\frac{1}{2} q_{1}=\frac{1}{2} V(\mathbf{p})+\frac{1}{2} V(\mathbf{q}) .
$$

However, A.3 does not hold: for $p>0$ and any $q$, we have $V(p, 0)=p>0=V(0, q)$.

\section{A. $4^{w}$ (Weak Consistency)}

Consider $V(p, q)=p^{2} q$. Then A.2 holds because $V(1,1)=1>0=V(0,0)$; A.3 holds because $V(p, 0)=0=V(0, q)$. And A. $5^{w}$ holds because the first mixed derivative of $V$ is positive, and hence $V$ is supermodular. But A.4 does not hold because $p V(1,1)+(1-$ p) $V(0,1)=p>p^{2}=V(p, 1)$.

\section{A.5 (Weak Complementarity)}

Consider the function

$$
V(p, q)= \begin{cases}\min \left(p, q, \frac{1}{3}, p+q-\frac{2}{3}\right) & \text { if } \frac{2}{3} \leq p+q \leq \frac{4}{3}, \\ \max (p+q-1,0) & \text { otherwise, }\end{cases}
$$

borrowed from Nelsen (2006, Exercise 2.11). Then A.2 holds because $V(1,1)=1>0=$ $V(0,0)$; A.3 holds because $V(p, 0)=0=V(0, q)$. And A. $4^{w}$ holds because $p V(1,1)+$ $(1-p) V(0,1)=p=V(p, 1)$, and similarly for the second relation. However, A. $5^{w}$ does not hold: let $\mathbf{p}=(1 / 3,2 / 3)$ and $\mathbf{q}=(2 / 3,1 / 3)$, so that $\mathbf{p} \vee \mathbf{q}=(2 / 3,2 / 3)$ and $\mathbf{p} \wedge \mathbf{q}=(1 / 3,1 / 3)$. Then $V(\mathbf{p} \vee \mathbf{q})+V(\mathbf{p} \wedge \mathbf{q})-V(\mathbf{p})-V(\mathbf{q})=-1 / 3<0$, contradicting supermodularity. 


\section{A.3. Proofs}

Proof of Theorem 1

Lemma A.1: Suppose that the ranking $\succeq$ satisfies A.1-A.2-A.3-A.4. Then

$$
(p, q) \sim p q(1,1) \oplus(1-p q)(0,0)
$$

for all $(p, q)$ in $[0,1]^{2}$.

Proof: By A.4, $(p, q) \sim p(1, q) \oplus(1-p)(0, q)$ and $(1, q) \sim q(1,1) \oplus(1-q)(1,0)$. By A.3, $(0, q) \sim(1,0) \sim(0,0)$. Using A.1 (mixture independence) repeatedly, we have $(p, q) \sim p(q(1,1) \oplus(1-q)(0,0)) \oplus(1-p)(0,0)=p q(1,1) \oplus(1-p q)(0,0) . \quad$ Q.E.D.

LEMMA A.2: If the ranking $\succeq$ satisfies A.1-A.2-A.3-A.4, then it satisfies A.5.

Proof: Consider A.5. Let $\mathbf{p} \bowtie \mathbf{q}$ in $[0,1]^{2}$. Assume without loss of generality $p_{1}>$ $q_{1}$ and $p_{2}<q_{2}$. Using Lemma A.1 and the reduction of compound lotteries implied by mixture independence (see, e.g., Property M5 in Fishburn, 1982, p. 11), we have

$$
\begin{aligned}
\frac{1}{2}(\mathbf{p} \vee \mathbf{q}) \oplus \frac{1}{2}(\mathbf{p} \wedge \mathbf{q})= & \frac{1}{2}\left(p_{1}, q_{2}\right) \oplus \frac{1}{2}\left(q_{1}, p_{2}\right) \\
\sim & \frac{1}{2}\left[p_{1} q_{2}(1,1) \oplus\left(1-p_{1} q_{2}\right)(0,0)\right] \\
& \oplus \frac{1}{2}\left[q_{1} p_{2}(1,1) \oplus\left(1-q_{1} p_{2}\right)(0,0)\right] \\
= & \left(\frac{p_{1} q_{2}+q_{1} p_{2}}{2}\right)(1,1) \oplus\left(1-\frac{p_{1} q_{2}+q_{1} p_{2}}{2}\right)(0,0) .
\end{aligned}
$$

Similarly, we find

$$
\frac{1}{2} \mathbf{p} \oplus \frac{1}{2} \mathbf{q} \sim\left(\frac{p_{1} p_{2}+q_{1} q_{2}}{2}\right)(1,1) \oplus\left(1-\frac{p_{1} p_{2}+q_{1} q_{2}}{2}\right)(0,0) .
$$

Moreover, $\left(p_{1}-q_{1}\right)\left(q_{2}-p_{2}\right)>0$ implies $p_{1} q_{2}+q_{1} p_{2}>p_{1} p_{2}+q_{1} q_{2}$. By A.2, we apply Theorem 4 in Herstein and Milnor (1953, p. 295) to obtain

$$
\begin{aligned}
& \left(\frac{p_{1} q_{2}+q_{1} p_{2}}{2}\right)(1,1) \oplus\left(1-\frac{p_{1} q_{2}+q_{1} p_{2}}{2}\right)(0,0) \\
& \quad \succ\left(\frac{p_{1} p_{2}+q_{1} q_{2}}{2}\right)(1,1) \oplus\left(1-\frac{p_{1} p_{2}+q_{1} q_{2}}{2}\right)(0,0)
\end{aligned}
$$

and thus

$$
\frac{1}{2}(\mathbf{p} \vee \mathbf{q}) \oplus \frac{1}{2}(\mathbf{p} \wedge \mathbf{q}) \succ \frac{1}{2} \mathbf{p} \oplus \frac{1}{2} \mathbf{q} .
$$

Theorem 1 . The ranking $\succeq$ on $[0,1]^{2}$ satisfies A.1-A.2-A.3-A.4 if and only if it is represented by the copula $\Pi(p, q)=p \cdot q$. 
ProOF: Necessity is obvious. We prove sufficiency. Clearly, A.4 implies A. ${ }^{w}$. Moreover, A.1-A.2-A.3-A.4 implies A.5 by Lemma A.2. Then Theorem 2 implies that $\succeq$ is represented by a unique copula $C(p, q)$. Using Property D.1 from Appendix A.1, $C(1, q)=q$ and $C(0, q)=0$ for any $q$ in $[0,1]$. Because A.4 (Consistency) requires

$$
p(1, q) \oplus(1-p)(0, q) \sim(p, q)
$$

for any $p, q$ in $[0,1]$, we obtain $C(p, q)=p C(1, q)+(1-p) C(0, q)=p q$.

Q.E.D.

\section{Proof of Theorem 2}

Theorem 2. The ranking $\succeq$ satisfies A.1-A.2-A.3-A. $4^{w}-\mathrm{A} .5^{w}$ if and only if it is represented by a (unique) copula $C:[0,1]^{2} \rightarrow[0,1]$.

ProOF: Necessity is obvious. We prove sufficiency. By A.1, the Mixture Space Theorem (Herstein and Milnor, 1953) implies that there exists a unique (up to increasing affine transformations) function $V:[0,1]^{2} \rightarrow \mathbb{R}$ that represents $\succeq$ and is linear with respect to $\oplus$. By A.2, $V(1,1)-V(0,0)>0$. Apply the appropriate increasing affine transformation and consider the (unique) function $C:[0,1]^{2} \rightarrow[0,1]$ defined by

$$
C(p, q)=\frac{V(p, q)-V(0,0)}{V(1,1)-V(0,0)} .
$$

We show that $C$ satisfies the two properties (D.1)-(D.2) given in Appendix A.1.

By A.3, we have $C(p, 0)=C(0, p)=C(0,0)=0$, for any $p$ in $[0,1]$. Moreover, clearly $C(1,1)=1$. By A. $4^{w}$ and linearity, for any $p$ in $[0,1]$, we get $C(p, 1)=C(p(1,1) \oplus(1-$ $p)(0,1))=p C(1,1)+(1-p) C(0,1)=p$; a similar argument shows that $C(1, p)=p$. This proves (D.1).

By A. $5^{w}$ and the linearity of $C$, for all $\mathbf{p}, \mathbf{q}$ in $[0,1]^{2}$, it follows that

$$
\frac{1}{2} C(\mathbf{p} \vee \mathbf{q})+\frac{1}{2} C(\mathbf{p} \wedge \mathbf{q})=C\left(\frac{1}{2}(\mathbf{p} \vee \mathbf{q}) \oplus \frac{1}{2}(\mathbf{p} \wedge \mathbf{q})\right) \geq C\left(\frac{1}{2} \mathbf{p} \oplus \frac{1}{2} \mathbf{q}\right)=\frac{1}{2} C(\mathbf{p})+\frac{1}{2} C(\mathbf{q}),
$$

so $C$ is supermodular and (D.2) holds.

\section{Proofs of Theorem 5 and 6}

The following lemma shows that A. $5^{w}$ is implied by A.1-A.2-A. $4^{w}-\mathrm{A} .7$. Given that A.7 also implies A.3, the proof of Theorem 5 is a special case of Theorem 2 and is omitted.

LEMMA A.3: If the ranking $\succeq$ satisfies A.1-A.2-A. $4^{w}-\mathrm{A} .7$, then A. $5^{w}$ holds.

PRoOF: Consider $\mathbf{p}=\left(p_{1}, p_{2}\right)$ and $\mathbf{q}=\left(q_{1}, q_{2}\right)$. If $\left(p_{1}-q_{1}\right)\left(p_{2}-q_{2}\right) \geq 0$, the left-hand side (LHS) and the right-hand side (RHS) in (4.1) are the same and A.5 ${ }^{w}$ holds trivially. In the following, suppose $p_{1}>q_{1}$ and $q_{2}>p_{2}$.

There are six possible cases. The first one occurs when $p_{2} \geq p_{1}$ and the second when $q_{1} \geq q_{2}$. We prove only the first case, because the argument for the second is analogous. The other four cases obtain when $p_{1}>p_{2}$ and $q_{2}>q_{1}$, from the combination of the two subcases $p_{1} \lessgtr q_{2}$ and the two subcases $p_{2} \lessgtr q_{1}$. We consider only the combination $p_{1}<q_{2}$ and $p_{2}>q_{1}$, because the argument for the other three is similar. 
Given $p_{2} \geq p_{1}$, we have $q_{2}>p_{2} \geq p_{1}>q_{1}$. By A.7, it follows $\mathbf{p} \vee \mathbf{q}=\left(p_{1}, q_{2}\right) \sim$ $\left(p_{1}, p_{1}\right) \sim\left(p_{1}, p_{2}\right)=\mathbf{p}$. Similarly, $\mathbf{p} \wedge \mathbf{q}=\left(q_{1}, p_{2}\right) \sim\left(q_{1}, q_{1}\right) \sim\left(q_{1}, q_{2}\right)=\mathbf{q}$. Then the LHS and the RHS in (4.1) are the same and A. $5^{w}$ holds.

Next, given $p_{1}<q_{2}$ and $p_{2}>q_{1}$, we have $q_{2}>p_{1}>p_{2}>q_{1}$. Applying A.7 thrice and A. $4^{w}$, we find $\mathbf{p} \vee \mathbf{q}=\left(p_{1}, q_{2}\right) \sim\left(p_{1}, p_{1}\right) \sim\left(p_{1}, 1\right) \sim p_{1}(1,1) \oplus\left(1-p_{1}\right)(0,1) \sim$ $p_{1}(1,1) \oplus\left(1-p_{1}\right)(0,0)$. Similarly, $\mathbf{p}=\left(p_{1}, p_{2}\right) \sim p_{2}(1,1) \oplus\left(1-p_{2}\right)(0,0)$. Moreover, $\mathbf{p} \wedge \mathbf{q} \sim\left(q_{1}, p_{2}\right) \sim\left(q_{1}, q_{2}\right)=\mathbf{q}$. Since $p_{1}>p_{2}$, Theorem 4 in Herstein and Milnor (1953, p. 295) implies $(1 / 2)(\mathbf{p} \vee \mathbf{q}) \oplus(1 / 2)(\mathbf{p} \wedge \mathbf{q}) \succ(1 / 2) \mathbf{p} \oplus(1 / 2) \mathbf{q}$.

Q.E.D.

We turn to the proof of Theorem 6 . A preliminary lemma shows that A. $5^{w}$ is implied by A.1-A.2-A.3-A. $4^{w}-\mathrm{A} .8$.

LEMMA A.4: If the ranking $\succeq$ satisfies A.1-A.2-A.3-A. $4^{w}-\mathrm{A} .8$, then A. $5^{w}$ holds.

PROOF: We start with four preliminary claims.

Claim 1. If $p_{1}+p_{2}<1$, then $\left(p_{1}, p_{2}\right) \sim(0,0)$. Using A.8 twice followed by A.3, $\left(p_{1}, p_{2}\right) \sim\left(\frac{p_{1}+p_{2}}{2}, \frac{p_{1}+p_{2}}{2}\right) \sim\left(p_{1}+p_{2}, 0\right) \sim(0,0)$ and the result follows.

Claim 2. If $p_{1}+p_{2} \geq 1$, then $\left(p_{1}, p_{2}\right) \sim\left(1, p_{1}+p_{2}-1\right)$. This follows immediately from A.8.

Claim 3. For any $\alpha, \beta \in[0,1],(1, \alpha) \succ(1, \beta)$ if and only if $\alpha>\beta$. Applying A. $4^{w}$ followed by A.1 and A.3, we have $(1, \alpha) \sim \alpha(1,1) \oplus(1-\alpha)(1,0) \sim \alpha(1,1) \oplus(1-\alpha)(0,0)$; the same holds for $(1, \beta)$. Because $(1,1) \succ(0,0)$ by A.2, the result follows from Theorem 4 in Herstein and Milnor (1953).

Claim 4. For any $\alpha, \beta \in[0,1], \frac{1}{2}(1, \alpha) \oplus \frac{1}{2}(1, \beta) \sim\left(1, \frac{\alpha+\beta}{2}\right)$. Using A.1 and A.4 ${ }^{w}$, $\frac{1}{2}(1, \alpha) \oplus \frac{1}{2}(1, \beta) \sim \frac{1}{2}[\alpha(1,1) \oplus(1-\alpha)(1,0)] \oplus \frac{1}{2}[\beta(1,1) \oplus(1-\beta)(1,0)]$. Because mixture independence implies the reduction of compound lotteries, the latter expression is equivalent to $\frac{\alpha+\beta}{2}(1,1) \oplus\left(1-\frac{\alpha+\beta}{2}\right)(1,0)$ and the result follows from A. $4^{w}$.

Having proved the claims, consider $\mathbf{p}=\left(p_{1}, p_{2}\right)$ and $\mathbf{q}=\left(q_{1}, q_{2}\right)$. Similarly to the proof of Lemma A.3, suppose $p_{1}>q_{1}$ and $q_{2}>p_{2}$. There are six possible cases. The first one occurs when $q_{1}+p_{2} \geq 1$ and the second when $p_{1}+q_{2} \leq 1$. We prove only the first case. The other four cases obtain when $q_{1}+p_{2}<1$ and $p_{1}+q_{2}>1$, from the combination of the two subcases $p_{1}+p_{2} \lessgtr 1$ and the two subcases $q_{1}+q_{2} \lessgtr 1$. We prove only the combination $p_{1}+p_{2}<1$ and $q_{1}+q_{2}>1$.

Given $q_{1}+p_{2} \geq 1$, Claim 2 implies $\mathbf{p} \vee \mathbf{q} \sim\left(1, p_{1}+q_{2}-1\right), \mathbf{p} \wedge \mathbf{q} \sim\left(1, q_{1}+p_{2}-1\right)$, $\mathbf{p} \sim\left(1, p_{1}+p_{2}-1\right)$, and $\mathbf{q} \sim\left(1, q_{1}+q_{2}-1\right)$. By Claim 4 ,

$$
(1 / 2)(\mathbf{p} \vee \mathbf{q}) \oplus(1 / 2)(\mathbf{p} \wedge \mathbf{q}) \sim\left(1, \frac{p_{1}+q_{1}+p_{2}+q_{2}-2}{2}\right) \sim(1 / 2) \mathbf{p} \oplus(1 / 2) \mathbf{q},
$$

so A. $5^{w}$ holds.

Next, given $p_{1}+p_{2}<1$ and $q_{1}+q_{2}>1$, Claim 1 and A.3 imply $\mathbf{p} \sim(\mathbf{p} \wedge \mathbf{q}) \sim(0,0) \sim$ $(1,0)$. Claim 2 gives $\mathbf{p} \vee \mathbf{q} \sim\left(1, p_{1}+q_{2}-1\right)$ and $\mathbf{q} \sim\left(1, q_{1}+q_{2}-1\right)$. By Claim $4,(1 / 2)(\mathbf{p} \vee$ $\mathbf{q}) \oplus(1 / 2)(\mathbf{p} \wedge \mathbf{q}) \sim\left(1, \frac{p_{1}+q_{2}-1}{2}\right)$ and $(1 / 2) \mathbf{p} \oplus(1 / 2) \mathbf{q} \sim\left(1, \frac{q_{1}+q_{2}-1}{2}\right)$. Because $p_{1}+q_{2}>$ $q_{1}+q_{2}$, applying Claim 3 yields $(1 / 2)(\mathbf{p} \vee \mathbf{q}) \oplus(1 / 2)(\mathbf{p} \wedge \mathbf{q}) \succ(1 / 2) \mathbf{p} \oplus(1 / 2) \mathbf{q} . \quad$ Q.E.D.

Theorem 6 . The ranking $\succeq$ satisfies A.1-A.2-A.3-A. $4^{w}-\mathrm{A} .8$ if and only if it is represented by the copula $W(p, q)=\max (p+q-1,0)$.

ProOF: Necessity is obvious. We prove sufficiency. Given Lemma A.4, Theorem 2 implies that $\succeq$ is represented by a unique copula $C\left(p_{1}, p_{2}\right)$. Because $W\left(p_{1}, p_{2}\right)$ is the only 
quasi-convex copula (see, for instance, Example 3.27 in Nelsen, 2006), it suffices to show that A.8 implies $C(\alpha \mathbf{p}+(1-\alpha) \mathbf{q}) \leq \max \{C(\mathbf{p}), C(\mathbf{q})\}$ for any $\alpha$ in $(0,1)$ and $\mathbf{p}, \mathbf{q}$ in $[0,1]^{2}$.

Assume without loss of generality $q_{1}+q_{2} \leq p_{1}+p_{2}$. Given $\mathbf{p}=\left(p_{1}, p_{2}\right)$, let $\overline{\mathbf{p}}=$ $\left(\frac{p_{1}+p_{2}}{2}, \frac{p_{1}+p_{2}}{2}\right)$ denote its symmetrization lying on the main diagonal. By A.8, $\mathbf{p} \sim \overline{\mathbf{p}} \mathrm{im}-$ plies $C(\mathbf{p})=C(\overline{\mathbf{p}})$. Because $C\left(p_{1}, p_{2}\right)$ is increasing on the main diagonal, we obtain

$$
C(\alpha \mathbf{p}+(1-\alpha) \mathbf{q})=C(\overline{\alpha \mathbf{p}+(1-\alpha) \mathbf{q}}) \leq C(\overline{\mathbf{p}})=C(\mathbf{p}),
$$

and thus $C$ is quasi-convex.

Q.E.D.

\section{Proof of Theorem 7}

For simplicity, we fix $i$ and drop subscripts when there is no risk of confusion; for instance, we write $\succeq$ instead of $\succeq_{k(i)}$ or $M$ instead of $M_{i}$.

LEMMA A.5: Given $p \in(0,1), L_{p_{n}} \rightarrow L_{p}$ in $A \cup \mathcal{L}$ if and only if $p_{n} \rightarrow p$.

PROOF: $(\Rightarrow)$ Let $\varepsilon>0$ be such that $(p-\varepsilon, p+\varepsilon) \subset[0,1]$. By B.4, the set $I=\{a \in$ $\left.A \cup \mathcal{L}: L_{p+\epsilon} \succ a \succ L_{p-\epsilon}\right\}$ is open because it is an intersection of open sets and $L_{p} \in I$ by B.3. Thus $L_{p_{n}} \rightarrow L_{p}$ implies that there exists $N$ such that $L_{p_{n}} \in I$ for all $n \geq N$; that is, $L_{p+\epsilon} \succ L_{p_{n}} \succ L_{p-\epsilon}$. Therefore, B.3 implies $p-\epsilon<p_{n}<p+\epsilon$ for all $n \geq N$, and thus $p_{n} \rightarrow p$.

$(\Leftarrow)$ It suffices to show that for any open neighborhood $\mathcal{O}$ of $L_{p}$, there is $N$ such that $L_{p_{n}} \in \mathcal{O}$ for all $n \geq N$. Let $\mathcal{O}$ be an open neighborhood of $L_{p}$; then $\mathcal{O} \cap \mathcal{L}$ is open in $\mathcal{L}$ since $\mathcal{O}$ is open in the disjoint union topology. But $p_{n} \rightarrow p$ implies $L_{p_{n}} \rightarrow L_{p}$ in $\mathcal{L}$, because $d\left(L_{p_{n}}, L_{p}\right)=\left|p_{n}-p\right| \rightarrow_{n} 0$. Therefore, there exists $N$ such that $L_{p_{n}} \in \mathcal{O} \cap \mathcal{L}$ for $n \geq N$, which implies $L_{p_{n}} \in \mathcal{O}$.

Q.E.D.

LEMMA A.6: For every $a \in A$, the set $W_{a}^{\mathcal{L}}=\left\{p \in[0,1]: L_{p} \prec a\right\}$ of strictly worse lotteries and the set $B_{a}^{\mathcal{L}}=\left\{p \in[0,1]: L_{p} \succ a\right\}$ of strictly better lotteries are open in the natural topology over $[0,1]$.

PRoOF: Fix $a \in A$. Clearly, $W_{a}^{\mathcal{L}}$ is open if and only if its complement $\bar{W}_{a}^{\mathcal{L}}$ is closed. Suppose by contradiction that $\bar{W}_{a}^{\mathcal{L}}$ is not closed. Then there exists a sequence $\left\{p_{n}\right\} \subset \bar{W}_{a}^{\mathcal{L}}$ such that $p_{n} \rightarrow p$ with $p \notin \bar{W}_{a}^{\mathcal{L}}$; then $p \in W_{a}^{\mathcal{L}}$ and thus $L_{p} \prec a$. However, $L_{p_{n}} \succeq a$ for all $n$ and, by Lemma A.5, $L_{p_{n}} \rightarrow L_{p}$ on $A \cup \mathcal{L}$. Hence, the set $W_{a}=\{y \in A \cup \mathcal{L}: y \succeq a\}$ is not closed in $A \cup \mathcal{L}$, contradicting B.4. The proof for $B_{a}^{\mathcal{L}}$ is analogous.

Q.E.D.

Theorem 7. Suppose that the preorder $\succeq_{k(i)}$ satisfies B.2-B.3-B.4. Then the benchmarking procedure uniquely extends the assessment $\hat{P}_{k(i)}$ on $\mathcal{L}_{i}$ to a continuous assessment $P_{k(i)}$ on $A \cup \mathcal{L}_{i}$ with $P_{k(i)}(\delta)=0$ and $P_{k(i)}\left(M_{i}\right)=1$, that represents $\succeq_{k(i)}$. Moreover, the restriction of $P_{k(i)}$ to $A$ is continuous and, if B.1 holds, also represents $\succsim_{i}$.

PROOF: We prove first that, for every $a$ in $A$, there exists a value $p$ such that $a \sim L_{p}$. Given $a$, the two open subsets $W_{a}^{\mathcal{L}}$ and $B_{a}^{\mathcal{L}}$ in $[0,1]$ are disjoint; therefore, the set $[0,1] \backslash$ $\left[W_{a}^{\mathcal{L}} \cup B_{a}^{\mathcal{L}}\right]$ cannot be empty because otherwise the connected interval $[0,1]$ would be covered by the disjoint union of two open sets. We pick a value $p$ from $[0,1] \backslash\left[W_{a}^{\mathcal{L}} \cup B_{a}^{\mathcal{L}}\right]$. Since $\succeq$ is a total preorder, we have $a \sim L_{p}$. 
Second, we show that such $p$ is unique. Suppose $[0,1] \backslash\left[W_{a}^{\mathcal{L}} \cup B_{a}^{\mathcal{L}}\right]$ contains two values $p>q$. By B.3, we would have $a \sim L_{p} \succ L_{q} \sim a$, contradicting the assumption that $\succeq$ is a preorder.

Define the extension $P$ on $A \cup \mathcal{L}$ by $P=\hat{P}$ on $\mathcal{L}$ and $P(a)=p$ when $a \sim L_{p}$ on $A$. We show that $P$ represents $\succeq$ on $A \cup \mathcal{L}$. Choose $x \succ y$ from $A \cup \mathcal{L}$ : if $P(y) \geq P(x)$, then B.3 would imply the contradiction $y \sim L_{P(y)} \succeq L_{P(x)} \sim x$. (Some equivalences in the last sentence hold as equalities if $x$ or $y$ are in $\mathcal{L}$.)

Finally, we prove that $P$ is continuous on $A \cup \mathcal{L}$. It suffices to show that the sets $W_{\alpha}=$ $\{x \in A \cup \mathcal{L}: P(x)<\alpha\}$ and $B_{\alpha}=\{x \in A \cup \mathcal{L}: P(x)>\alpha\}$ are open for every $\alpha$ in [0,1]. Consider only $W_{\alpha}$, because the argument for $B_{\alpha}$ is analogous. Clearly, $W_{0}=\emptyset$ is open. So assume $\alpha$ in $(0,1]$. Then

$$
W_{\alpha}=\{x \in A \cup \mathcal{L}: P(x)<\alpha\}=\left\{x \in A \cup \mathcal{L}: P(x)<P\left(L_{\alpha}\right)\right\}=\left\{x \in A \cup \mathcal{L}: x \prec L_{\alpha}\right\}
$$

is an open set in $A \cup \mathcal{L}$ by B.4.

Q.E.D.

\section{A.4. Consistency With the Ordinal Nash Solution}

We consider a bargaining problem with ordinal preferences, assuming for simplicity that there is an alternative $a^{\circ} \succ_{i} \delta$ for both $i$.

Let $L_{p}(a)$ denote the elementary lottery $p a \oplus(1-p) \delta$ between an alternative $a$ in $A$ and the default outcome $\delta$. Consider the set $\mathcal{L}(A \mid \delta)=\{p a \oplus(1-p) \delta: a \in A, p \in[0,1]\}$ of the elementary lotteries and note that $\mathcal{L}(A \mid \delta) \subseteq \mathcal{L}(A)$. Grant and Kajii (1995) assumed that preferences are defined over $\mathcal{L}(A)$, but they observed that the ordinal Nash outcome requires only that bargainers have preferences on $\mathcal{L}(A \mid \delta)$. Our mild generalization concerns this latter case.

We assume that each bargainer $i$ has a preference relation $\succsim_{i}$ over $\mathcal{L}(A \mid \delta)$ such that:

R.1: $a \sim_{i} L_{1}(a)$ for any $a$;

R.2: $q L_{p}\left(M_{i}\right) \oplus(1-q) \delta \sim_{i} L_{p q}\left(M_{i}\right)$ for any $p, q$;

R.3: the sets $\left\{p \in[0,1]: L_{p}\left(M_{i}\right) \succ_{i} L_{q}(a)\right\}$ and $\left\{p \in[0,1]: L_{q}(a) \succ_{i} L_{p}\left(M_{i}\right)\right\}$ are open in the usual topology for any $q$ and for any $a$.

The first property is standard and embeds $A$ in $\mathcal{L}(A \mid \delta)$. The other two restrict to $\mathcal{L}(A \mid \delta)$ the reduction of compounded lotteries and the continuity assumed by Grant and Kajii (1995) for $\mathcal{L}(A)$.

The following key properties are mild relaxations of the DOM and WH assumptions in Grant and Kajii (1995).

$\mathrm{D}^{w}$-Stochastic dominance: For any $a$ in $A$, if $p>q$, then $L_{p}(a) \succ_{i} L_{q}(a)$.

$\mathrm{H}^{w}$-Homogeneity: If $a \sim_{i} L_{q}\left(M_{i}\right)$, then $L_{p}(a) \sim_{i} L_{p q}\left(M_{i}\right)$.

Our first lemma states the existence of a function $P_{i}$ on $\mathcal{L}(A \mid \delta)$ that represents $i$ 's preferences. After noting that R.3 assumes the content of Lemma A.6, R.1 implies B.2 and $\mathrm{D}^{w}$ implies B.3, its proof is analogous to the proof of Theorem 7.

Lemma A.7: A preference relation $\succsim_{i}$ satisfies $R .1, R .3$, and $D^{w}$ if and only if there is a function $P_{i}$ that represents $\succsim_{i}$ on $\mathcal{L}(A \mid \delta)$ such that $P_{i}(\delta)=0, P_{i}\left(M_{i}\right)=1$, and $P_{i}$ is linear w.r.t. $\oplus$ over $\mathcal{L}_{i}$.

The next lemma is the equivalent of Lemma 1 in Grant and Kajii (1995, p. 1243). 
LEMmA A.8: Under $H^{w}$, the function $P_{i}$ is linear w.r.t. $\oplus$ over $\mathcal{L}(A \mid \delta)$; that is, it has a $D L$ representation.

PRoOF: For $a$ in $A$ and $p$ in [0,1], we show that $P_{i}\left(L_{p}(a)\right)=p P_{i}(a)+(1-p) P_{i}(\delta)=$ $p P_{i}(a)$. By the linearity of $P_{i}$ over $\mathcal{L}_{i}, a \sim_{i} L_{P_{i}(a)}\left(M_{i}\right)$. By $\mathrm{H}^{w}, L_{p}(a) \sim_{i} L_{p P_{i}(a)}\left(M_{i}\right)$. Write $P$ for $P_{i}\left(L_{p}(a)\right)$ and consider the lottery $P M_{i} \oplus(1-P) \delta$ in $\mathcal{L}_{i}$. By the linearity of $P_{i}$ over $\mathcal{L}_{i}, P_{i}\left[P M_{i} \oplus(1-P) \delta\right]=P$. Because $P_{i}(\cdot)$ represents $\succsim_{i}$ on $\mathcal{L}(A \mid \delta)$, this implies $L_{P}\left(M_{i}\right) \sim_{i} L_{p}(a)$. Then transitivity implies $L_{P}\left(M_{i}\right) \sim_{i} L_{p P_{i}(a)}\left(M_{i}\right)$. Finally, $\mathrm{D}^{w}$ yields $P_{i}\left(L_{p}(a)\right)=p P_{i}(a)$.

We are ready to prove the consistency between the Nash copula and the ordinal Nash solution. This result mirrors Lemma 2 in Grant and Kajii (1995, p. 1243).

Proposition A.9: Under $D^{w}$ and $H^{w}$, an alternative $a^{*}$ is an ordinal Nash solution if and only if it maximizes the Nash copula $P_{1}(a) \cdot P_{2}(a)$.

PROOF: Sufficiency. Let $a^{*} \in \arg \max _{a \in A} P_{1}(a) P_{2}(a)$; that is, $P_{1}\left(a^{*}\right) P_{2}\left(a^{*}\right) \geq P_{1}(a) P_{2}(a)$ for any $a$ in $A$. In particular, note that $P_{1}\left(a^{*}\right) P_{2}\left(a^{*}\right) \geq P_{1}\left(a^{\circ}\right) P_{2}\left(a^{\circ}\right)>P_{1}(\delta) P_{2}(\delta)=0$. Let $p$ be in $(0,1]$ and $a$ in $A$. Suppose $L_{p}(a) \succ_{1} a^{*}$. Because $P_{1}$ represents $\succsim_{1}$ by Lemma A.7 and has a DL representation on $\mathcal{L}(A \mid \delta)$ by Lemma A.8, we have $p P_{1}(a)>P_{1}\left(a^{*}\right)>0$ and thus $P_{1}(a)>0$. Hence,

$$
p P_{1}(a) P_{2}\left(a^{*}\right)>P_{1}\left(a^{*}\right) P_{2}\left(a^{*}\right) \geq P_{1}(a) P_{2}(a)
$$

implies $p P_{2}\left(a^{*}\right)>P_{2}(a)$, and thus $L_{p}\left(a^{*}\right) \succ_{2} a$.

Necessity. Suppose that $a^{*}$ is an ordinal Nash solution but does not maximize the Nash copula. Then there exists some $a$ in $A$ such that $P_{1}(a) P_{2}(a)>P_{1}\left(a^{*}\right) P_{2}\left(a^{*}\right)>0$. We can find $p$ in $(0,1)$ such that $\frac{P_{2}(a)}{P_{2}\left(a^{*}\right)}>p>\frac{P_{1}\left(a^{*}\right)}{P_{1}(a)}$. Then $p P_{1}(a)>P_{1}\left(a^{*}\right)$ and $P_{2}(a)>p P_{2}\left(a^{*}\right)$ imply $L_{p}(a) \succ_{1} a^{*}$ and $a \succ_{2} L_{p}\left(a^{*}\right)$, contradicting the assumption that $a^{*}$ is an ordinal Nash solution.

Q.E.D.

\section{A.5. The Nash Product With $n \geq 2$ Bargainers}

Consider a ranking $\succeq$ over all $n$-tuples of acceptance probabilities in $[0,1]^{n}$. Let $\left(p, p_{-i}\right)$ denote the $n$-tuple $\left(p_{1}, \ldots, p_{i-1}, p, p_{i+1}, \ldots, p_{n}\right)$ with $p_{i}=p$. We state the analogs of the axioms for the case with two bargainers discussed in Section 3. Then we prove an extension of Theorem 1 to $n$ bargainers.

A.1-Regularity: $\succeq$ is a complete preorder, continuous and mixture independent.

A.2-Non-triviality: $(1, \ldots, 1) \succ(0, \ldots, 0)$.

A.3-Disagreement indifference: For any $i, j$ and any $p_{-i}, q_{-j},\left(0, p_{-i}\right) \sim\left(0, q_{-j}\right)$.

A.4-Consistency: For any $i$ and any $p \in[0,1], p\left(1, p_{-i}\right) \oplus(1-p)\left(0, p_{-i}\right) \sim\left(p, p_{-i}\right)$.

THEOREM 10: The ranking $\succeq$ on $[0,1]^{n}$ satisfies A.1-2-3-4 if and only if it is represented by the product copula $C\left(p_{1}, \ldots, p_{n}\right)=p_{1} \cdots p_{n}$. 
ProOF: Necessity is obvious. We prove sufficiency. By A.1 and the Mixture Space Theorem, there exists a unique (up to increasing affine transformations) function $V$ : $[0,1]^{n} \rightarrow \mathbb{R}$ that represents $\succeq$ and is linear with respect to mixtures. By A.2, $V(1, \ldots, 1)-$ $V(0, \ldots, 0)>0$. Apply the appropriate increasing affine transformation and consider the (unique) function $C:[0,1]^{n} \rightarrow[0,1]$ defined by

$$
C\left(p_{1}, \ldots, p_{n}\right)=\frac{V\left(p_{1}, \ldots, p_{n}\right)-V(0, \ldots, 0)}{V(1, \ldots, 1)-V(0, \ldots, 0)} .
$$

By A.3, we have $C\left(0, p_{-i}\right)=C\left(0, q_{-j}\right)=C(0, \ldots, 0)=0$ for any $i, j$ : we say that $C$ is grounded. Moreover, $C(1, \ldots, 1)=1$.

Given a vector $\left(p_{1}, \ldots, p_{n}\right) \in[0,1]^{n}$, we have $p_{1}\left(1, p_{-1}\right) \oplus\left(1-p_{1}\right)\left(0, p_{-1}\right) \sim\left(p_{1}, p_{-1}\right)$ by A.4. Moreover, $C\left(p_{1}, \ldots, p_{n}\right)=p_{1} C\left(1, p_{-1}\right)+\left(1-p_{1}\right) C\left(0, p_{-1}\right)=p_{1} C\left(1, p_{-1}\right)$ because $C$ is linear and grounded. Apply A.4 again to the second component of the $n$-tuple $\left(1, p_{-1}\right)=\left(1, p_{2}, \ldots, p_{n}\right)=\left(1, p_{2}, p_{-12}\right)$ and obtain $p_{2}\left(1,1, p_{-12}\right) \oplus\left(1-p_{2}\right)\left(1,0, p_{-12}\right) \sim$ $\left(1, p_{2}, p_{-12}\right)$; this yields $C\left(1, p_{2}, p_{-12}\right)=p_{2} C\left(1,1, p_{-12}\right)+\left(1-p_{2}\right) C\left(1,0, p_{-12}\right)=p_{2} C(1$, $\left.1, p_{-12}\right)$ because $C$ is linear and grounded. Thus, $C\left(p_{1}, \ldots, p_{n}\right)=p_{1} C\left(1, p_{-1}\right)=p_{1} p_{2} \times$ $C\left(1,1, p_{-12}\right)$. Iteration leads to $C\left(p_{1}, \ldots, p_{n}\right)=p_{1} \cdots p_{n} \cdot C(1, \ldots, 1)=p_{1} \cdots$ $p_{n}$.

Q.E.D.

\section{REFERENCES}

Alvoni, E., AND P. L. PAPINI (2007): "Quasi-Concave Copulas, Asymmetry and Transformations," Commentationes MathematicaUniversitatis Carolinae, 48, 311-319. [856]

ANBAR, D., AND E. KALAI (1978): "A One-Shot Bargaining Problem," International Journal of Game Theory, 7, 13-18. [839]

ARRow, K. J. (1951): Social Choice and Individual Values New York: Wiley. [847]

Blackorby, C., W. BosserT, AND D. DonALDSON (1994): "Generalized Ginis and Cooperative Bargaining Solutions," Econometrica, 62, 1161-1178. [848,849]

Bleichrodt, H., C. LI, I. Moscati, AND P. P. WAKKer (2016): "Nash Was a First to Axiomatize Expected Utility," Theory and Decision, 81, 309-312. [840]

BORDER, K., AND U. SEgAL (1997): "Preferences Over Solutions to the Bargaining Problem," Econometrica, 65, 1-18. [843,851]

CASTAGnoli, E., AND M. LiCAlzi (1996): “Expected Utility Without Utility,” Theory and Decision, 41, 281301. [851]

DHILlON, A., AND J.-F. MERTENS (1999): “Relative Utilitarianism,” Econometrica, 67, 471-498. [847]

FISHBURN, P. (1970): Utility Theory for Decision Making. New York: Wiley. [842] (1982): The Foundations of Expected Utility. Dordrecht: Reidel. [858]

FRANCETICH, A. (2013): "Notes on Supermodularity and Increasing Differences in Expected Utility," Economics Letters, 121, 206-209. [845]

GRANT, S., AND A. KAJII (1995): "A Cardinal Characterization of the Rubinstein-Safra-Thomson Axiomatic Bargaining Theory," Econometrica, 63, 1241-1249. [853,862,863]

HARSANYI, J. C. (1956): "Approaches to the Bargaining Problem Before and After the Theory of Games: A Critical Discussion of Zeuthen's, Hicks', and Nash's Theories," Econometrica, 24, 144-157. [850]

_ (1962): "Bargaining in the Ignorance of the Opponent's Utility Function," Journal of Conflict Resolution, 6, 29-38. [850,852]

HARSANYI, J. C., AND R. SElten (1972): "A Generalized Nash Solution for Two-Person Bargaining Games With Incomplete Information,” Management Science, 18, 80-106. [855]

HersteIn, I. N., AND J. MiLnOR (1953): "An Axiomatic Approach to Measurable Utility,” Econometrica, 21, 291-297. [842,858-860]

KALAI, E. (1977): "Proportional Solutions to Bargaining Situations: Interpersonal Utility Comparisons," Econometrica, 45, 1623-1630. [847]

KALAI, E., AND M. SMORODINSKY (1975): “Other Solutions to Nash's Bargaining Problem,” Econometrica, 43, 513-518. [853] 
KANEKO, M., AND K. NAKAmURA (1979): “The Nash Social Welfare Function,” Econometrica, 47, 423-435. [851]

KIHLSTROM, R., A. E. Roth, AND D. SCHMEIDLER (1981): "Risk Aversion and Solutions to Nash's Bargaining Problem," in Game Theory and Mathematical Economics, ed. by O. Moeschlin and D. Pallaschke. Amsterdam: North-Holland, 65-71. [854]

LiCALZI, M. (1999): "A Language for the Construction of Preferences Under Uncertainty," Revista de la Real Academia de Ciencias Exactas, Fìsicas y Naturales, 93, 439-450. [840,855]

LIEBSCHER, E. (2008): "Construction of Asymmetric Multivariate Copulas," Journal of Multivariate Analysis, 99, 2234-2250. [855]

Mariotti, M., AND R. Veneziani (2018): "Opportunities as Chances: Maximising the Probability That Everybody Succeeds," Economic Journal, 128, 1609-1633. [851]

MiYaGaWA, E. (2002): "Subgame-Perfect Implementation of Bargaining Solutions," Games and Economic Behavior, 41, 292-308. [852]

NASH, J. F. (1950): “The Bargaining Problem,” Econometrica, 18, 155-162. [837,839,840,842,848,850] (1953): "Two-Person Cooperative Games," Econometrica, 21, 128-140. [850]

NELSEN, R. B. (2006): An Introduction to Copulas (Second Ed.). New York: Springer. [848,856,857,861]

- (2007): "Extremes of Nonexchangeability," Statistical Papers, 48, 329-336. [856]

PETERS, H., AND P. WAKKER (1991): "Independence of Irrelevant Alternatives and Revealed Group Preferences," Econometrica, 59, 1787-1801. [853]

RoTH, A. E. (1979): Axiomatic Models of Bargaining. Berlin: Springer. 1979. [839]

RUBINSTEIN, A., Z. SAFRA, AND W. THOMSON (1992): "On the Interpretation of the Nash Bargaining Solution and Its Extension to Non-Expected Utility Preferences," Econometrica, 60, 1171-1186. [837,840,852]

SHALEV, J. (2002): "Loss Aversion and Bargaining," Theory and Decision, 52, 201-232. [855]

SKLAR, A. (1959): "Fonctions de répartition à $n$ dimensions et leurs marges," Publications de l'Institut de Statistique de l'Université de Paris, 8, 229-231. [856]

Subramanian, G. (2010): Negotiauctions. New York: Norton. [843]

Thomson, W. (1994): “Cooperative Models of Bargaining," in Handbook of Game Theory, Vol. 2, ed. by R. J. Aumann and S. Hart. Amsterdam: North-Holland, 1237-1284. [837]

TROCKEL, W. (2008): "The Nash Product Is a Utility Representation of the Pareto Ordering," Economics Letters, 99, 220-222. [839]

Valenciano, F., AND J. M. Zarzuelo (1997): “On Nash's Hidden Assumption,” Games and Economic Behavior, 21, 266-281. [842]

Voorneveld, M. (2014): “From Preferences to Leontief Utility,” Economic Theory Bulletin, 2, 197-204. [847]

\section{Co-editor Itzhak Gilboa handled this manuscript.}

Manuscript received 24 July, 2015; final version accepted 27 January, 2019; available online 29 January, 2019. 